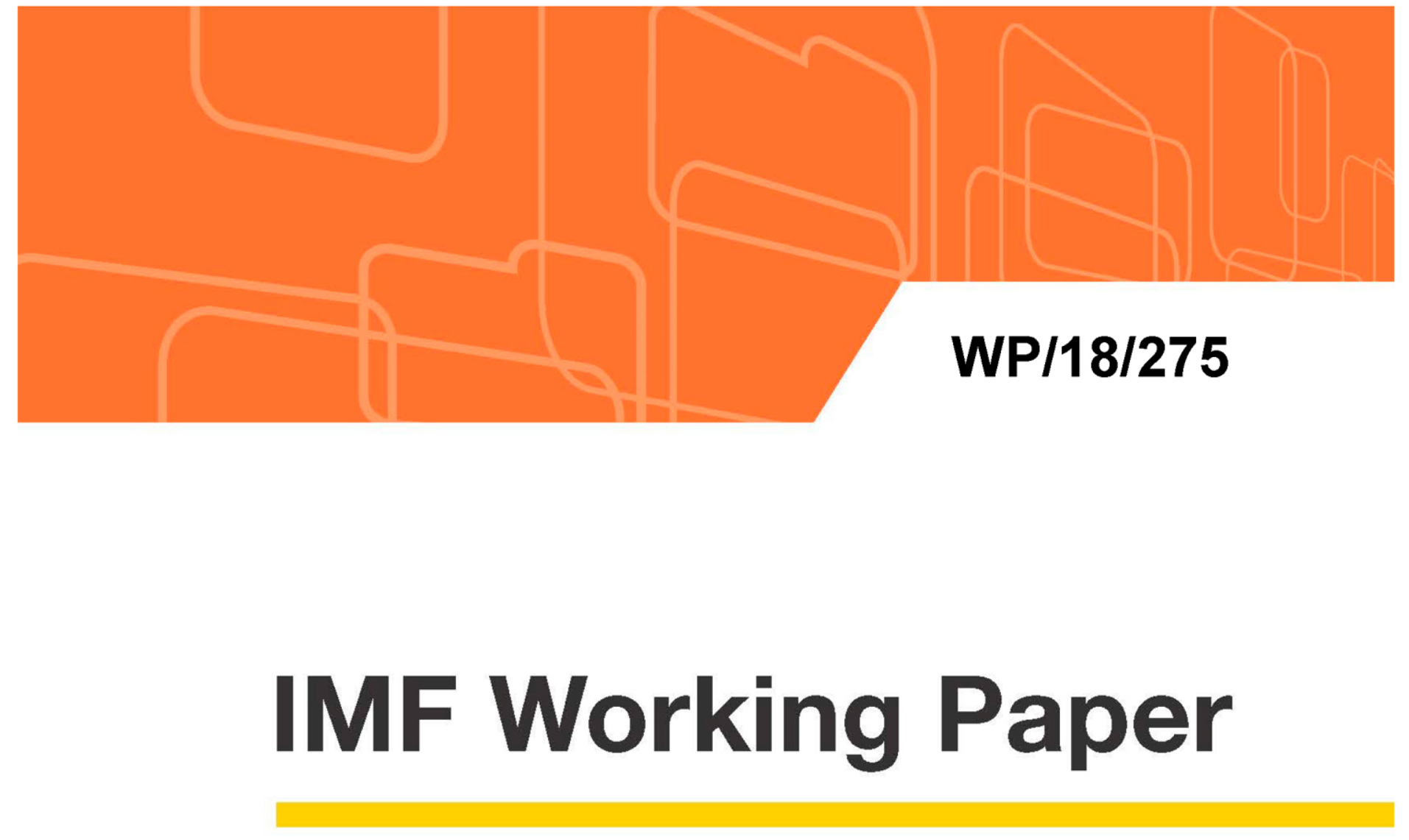

\title{
A Closed Form Multivariate Linear Filter
}

by Francis Vitek

IMF Working Papers describe research in progress by the author(s) and are published to elicit comments and to encourage debate. The views expressed in IMF Working Papers are those of the author(s) and do not necessarily represent the views of the IMF, its Executive Board, or IMF management. 


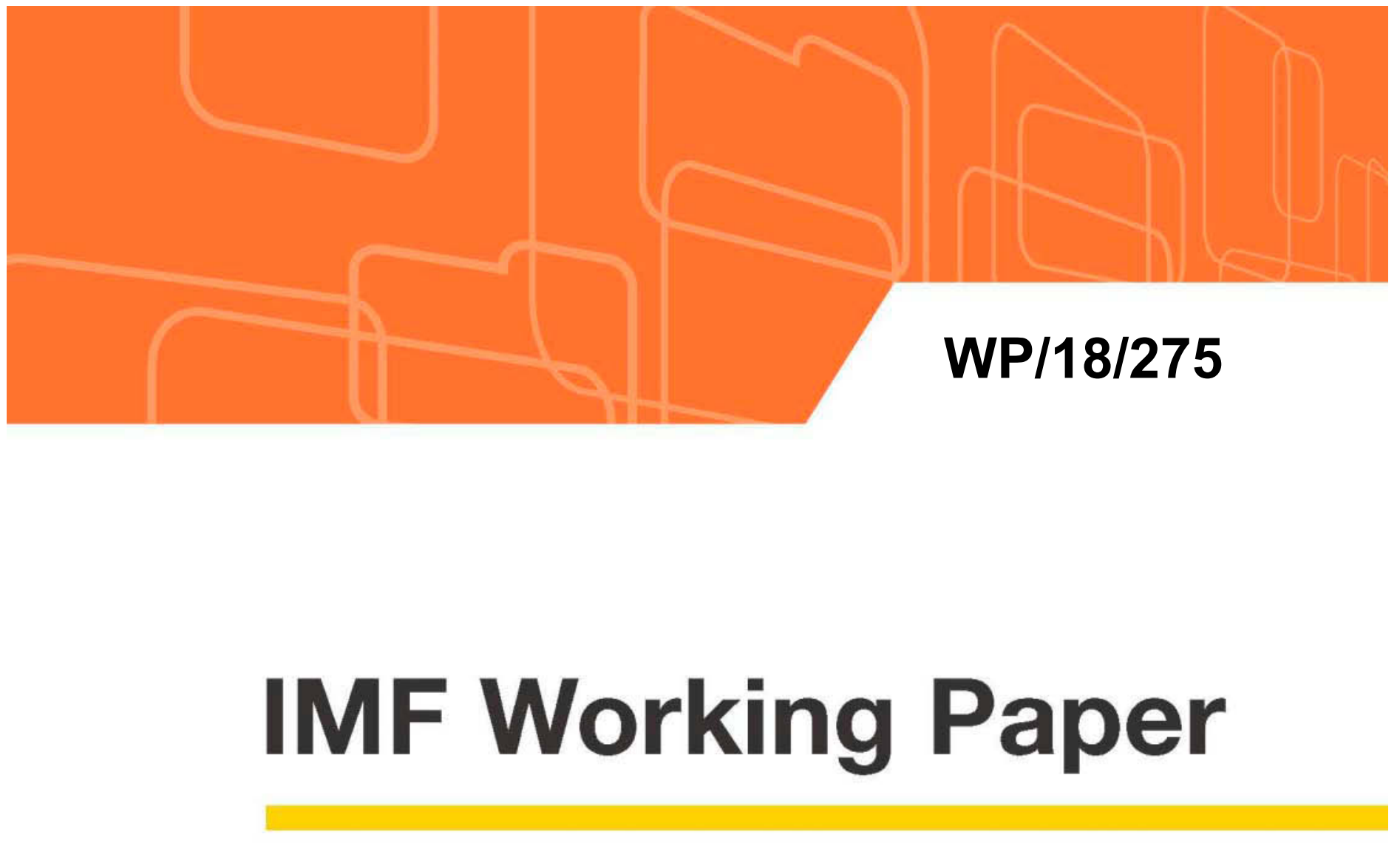

\section{A Closed Form Multivariate Linear Filter}

by Francis Vitek

IMF Working Papers describe research in progress by the author(s) and are published to elicit comments and to encourage debate. The views expressed in IMF Working Papers are those of the author(s) and do not necessarily represent the views of the IMF, its Executive Board, or IMF management. 


\title{
IMF Working Paper
}

Monetary and Capital Markets Department

\section{A Closed Form Multivariate Linear Filter}

Prepared by Francis Vitek ${ }^{1}$

Authorized for distribution by Ulric Eriksson von Allmen

December 2018

IMF Working Papers describe research in progress by the author(s) and are published to elicit comments and to encourage debate. The views expressed in IMF Working Papers are those of the author(s) and do not necessarily represent the views of the IMF, its Executive Board, or IMF management.

\begin{abstract}
This paper considers the problem of jointly decomposing a set of time series variables into cyclical and trend components, subject to sets of stochastic linear restrictions among these cyclical and trend components. We derive a closed form solution to an ordinary problem featuring homogeneous penalty term difference orders and static restrictions, as well as to a generalized problem featuring heterogeneous penalty term difference orders and dynamic restrictions. We use our Generalized Multivariate Linear Filter to jointly estimate potential output, the natural rate of unemployment and the natural rate of interest, conditional on selected equilibrium conditions from a calibrated New Keynesian model.
\end{abstract}

JEL Classification Numbers: C32; E32; E52

Keywords: Closed form; Multivariate linear filter; New Keynesian model; Potential output; Natural rate of unemployment; Natural rate of interest

Author's E-Mail Address: FVitek@imf.org

\footnotetext{
${ }^{1}$ The author gratefully acknowledges advice provided by Tobias Adrian, as well as comments and suggestions received from many colleagues at the IMF.
} 


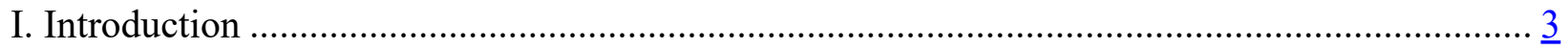

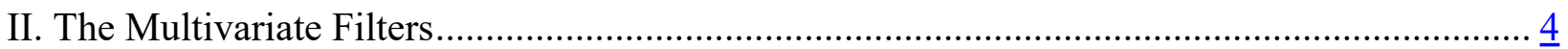

A. The Ordinary Multivariate Linear Filter .................................................................. 4

B. The Generalized Multivariate Linear Filter …............................................................. 5

III. The New Keynesian Model .................................................................................

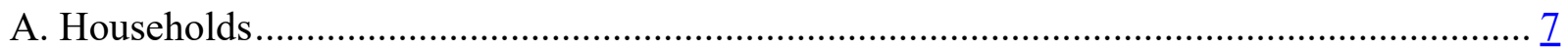

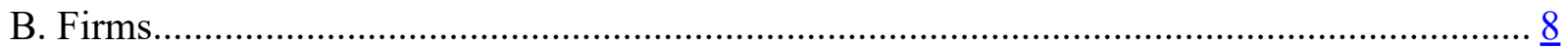

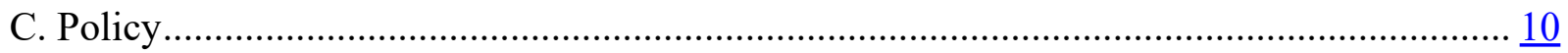

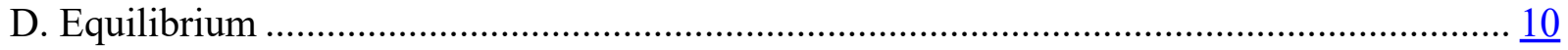

IV. Estimation of Potential Output and Natural Rates.................................................... 12

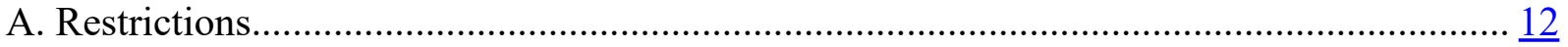

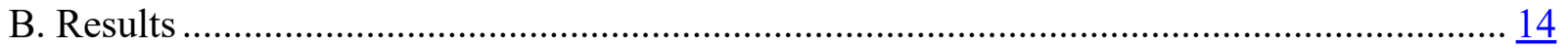

Figure 1 . Estimated Trend Components from Multivariate versus Univariate Filters ........ 15

Figure 2 . Estimated Cyclical Components from Multivariate versus Univariate Filters..... $\underline{16}$

C. Robustness ..................................................................................................... 16

Table 1 . Sensitivity of Estimation Results to Smoothing Parameter Perturbations............. 17

Table 2 . Sensitivity of Estimation Results to Weight Parameter Perturbations ................. 18

Table 3 . Sensitivity of Estimation Results to Structural Parameter Perturbations ............. $\underline{18}$

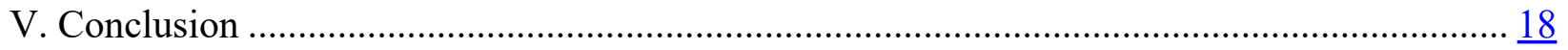

Appendix A . Proof of Proposition 1 ............................................................................... 18

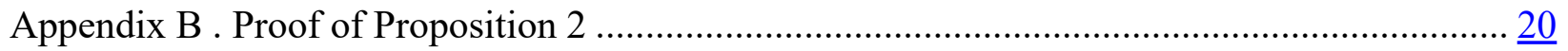

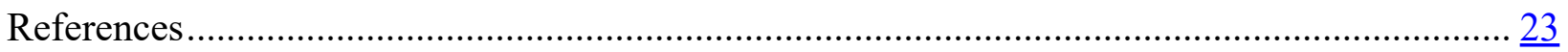




\section{INTRODUCTION}

Unobserved variables are central to monetary economics, which conventionally treats the monetary transmission mechanism as a cyclical phenomenon empowered by nominal rigidities. Indeed, unobserved indicators of inflationary pressure such as the output and unemployment rate gaps, and measures of the stance of monetary policy such as the interest rate gap, are key inputs into the conduct of monetary policy under an inflation targeting regime. These gap variables are measured relative to potential output, the natural rate of unemployment and the natural rate of interest, respectively.

Closed form univariate linear filters, such as those associated with Hodrick and Prescott (1997) and Lucas (1980), remain benchmark estimators of potential output, the natural rate of unemployment and the natural rate of interest, due to their computational simplicity and accordance with the widespread belief among monetary policymakers that these unobserved variables evolve smoothly. But these closed form univariate linear filters are routinely criticized as being atheoretic, as they do not account for dynamic interrelationships that are theoretically predicted to hold among such unobserved variables. Moreover, their statistical properties have been criticized by King and Rebelo (1993), Harvey and Jaeger (1993), Phillips and Jin (2015), and Hamilton (2017). To address these critiques, linear unobserved components models are commonly used to capture dynamic interrelationships among cyclical and trend components. Influential papers that jointly estimate potential output, the natural rate of unemployment or the natural rate of interest within an unobserved components framework include Clark (1989), Apel and Jansson (1999), and Laubach and Williams (2003). But full information maximum likelihood estimation of linear unobserved components models, facilitated by the recursive multivariate linear filter due to Kalman (1960), can be computationally complex, yielding cyclical and trend component estimates that can be sensitive to model specification choices and initial conditions.

This paper considers the problem of jointly decomposing a set of time series variables into cyclical and trend components, subject to sets of stochastic linear restrictions among these cyclical and trend components. We derive a closed form solution to an ordinary problem featuring homogeneous penalty term difference orders and static linear restrictions, as well as to a generalized problem featuring heterogeneous penalty term difference orders and dynamic linear restrictions. The resultant Ordinary Multivariate Linear Filter (OMLF) or Generalized Multivariate Linear Filter (GMLF) nests the closed form univariate linear filters associated with Hodrick and Prescott (1997) and Lucas (1980), while selectively accounting for static or dynamic interrelationships among variables of the form incorporated into linear unobserved components models, respectively. These closed form multivariate linear filters do not depend on initial conditions, as they operate over the entire sample in one step, unlike recursive multivariate linear filters which pass sequentially through the sample. We use our GMLF to jointly estimate potential output, the natural rate of unemployment and the natural rate of interest for the United States, conditional on selected approximate linear equilibrium conditions from a calibrated New

Keynesian model disciplining dynamic interrelationships among these unobserved variables. The 
estimates exhibit economically significant and interpretable deviations from those generated using the corresponding univariate filters, the sources of which are revealed by sensitivity analysis.

As hybrids of closed form univariate linear filters and linear unobserved components models, our closed form multivariate linear filters inherit properties of both, to varying degrees depending on the calibration of the tuning parameters that enter into the objective function that they minimize. A related hybrid approach to estimating unobserved components was proposed by Laxton and Tetlow (1992), who numerically extend the filter associated with Hodrick and Prescott (1997) to condition on a set of stochastic linear restrictions into which the cyclical component of the variable under consideration enters. In contrast, this paper analytically derives solutions to multivariate as opposed to univariate minimization problems that condition on sets of stochastic linear restrictions among both cyclical and trend components. Another related hybrid approach was proposed by Vitek (2009), who instead estimates a linear unobserved components model conditional on judgment concerning the paths of trend components generated using the filter associated with Hodrick and Prescott (1997), by augmenting its linear state space representation with a set of stochastic linear restrictions on selected state variables.

The organization of this paper is as follows. The next section presents the multivariate filters. The following section develops the New Keynesian model. The joint estimation of potential output and natural rates is the subject of section four. Finally, section five offers conclusions and recommendations for further research.

\section{The Multivariate Filters}

Consider a vector stochastic process $\left\{\left\{y_{i, t}\right\}_{i=1}^{N}\right\}_{t=1}^{T}$ of dimension $N$ that is observed for $T$ periods. Suppose that this vector stochastic process is additively separable into cyclical and trend components, that is $y_{i, t}=\hat{y}_{i, t}+\bar{y}_{i, t}$.

\section{A. The Ordinary Multivariate Linear Filter}

We define the Ordinary Multivariate Linear Filter as that trend component estimator $\left\{\left\{\bar{y}_{i, t \mid T}\right\}_{i=1}^{N}\right\}_{t=1}^{T}$ which minimizes objective function:

$$
S\left(\left\{\left\{\bar{y}_{i, t}\right\}_{i=1}^{N}\right\}_{t=1}^{T}\right)=\sum_{i=1}^{N} \sum_{t=1}^{T} \hat{y}_{i, t}^{2}+\lambda^{2} \sum_{i=1}^{N} \sum_{t=d+1}^{T}\left(\Delta^{d} \bar{y}_{i, t}\right)^{2}+\gamma^{2} \sum_{g=1}^{G} \sum_{t=1}^{T}\left(\sum_{i=1}^{N} \phi_{g, i} \hat{y}_{i, t}\right)^{2}+\psi^{2} \sum_{h=1}^{H} \sum_{t=1}^{T}\left(\sum_{i=1}^{N} \theta_{h, i} \bar{y}_{i, t}\right)^{2} .
$$

This minimization problem strikes a balance between minimizing the sum of squares of the cyclical components and the sum of squares of the ordinary difference of order $d$ of the trend components. As $\lambda$ increases, the estimated trend components become smoother, converging to deterministic polynomials of degree $d-1$ in the limit as $\lambda \rightarrow \infty$. We therefore recommend choosing the minimum value of $d$ for which $\left\{\Delta^{d-1} y_{i, t}\right\}_{t=d}^{T}$ does not exhibit a long run trend for all $i=1, \ldots, N$. This minimization problem also quadratically penalizes deviations of $G$ linearly independent static linear combinations of the cyclical components and $H$ linearly independent 
static linear combinations of the trend components from zero, where $G \leq N$ and $H \leq N$. As $\gamma$ or $\psi$ increases, the estimated cyclical or trend components more closely satisfy the stochastic linear restrictions imposed on their comovement, which become deterministic in the limit as $\gamma \rightarrow \infty$ or $\psi \rightarrow \infty$, respectively. In the univariate case, this minimization problem reduces to that considered by Hodrick and Prescott (1997) for $d=2$, and to that considered by Lucas (1980) for $d=1$, given that $\gamma=\psi=0$.

Proposition 1. Let $\overline{\boldsymbol{Y}}_{T}$ denote the Ordinary Multivariate Linear Filter. Using matrix notation, objective function (1) may be expressed as

$$
S(\overline{\boldsymbol{Y}})=\operatorname{Tr}\left(\hat{\boldsymbol{Y}}^{\top} \hat{\boldsymbol{Y}}\right)+\lambda^{2} \operatorname{Tr}\left(\left(\boldsymbol{\Delta}^{d} \overline{\boldsymbol{Y}}\right)^{\top}\left(\boldsymbol{\Delta}^{d} \overline{\boldsymbol{Y}}\right)\right)+\gamma^{2} \operatorname{Tr}\left((\hat{\boldsymbol{Y}} \boldsymbol{\Phi})^{\top}(\hat{\boldsymbol{Y}} \boldsymbol{\Phi})\right)+\psi^{2} \operatorname{Tr}\left((\overline{\boldsymbol{Y}} \boldsymbol{\Theta})^{\top}(\overline{\boldsymbol{Y}} \boldsymbol{\Theta})\right),
$$

where

$$
\boldsymbol{Y}=\left[\begin{array}{ccc}
y_{1,1} & \cdots & y_{N, 1} \\
\vdots & \ddots & \vdots \\
y_{1, T} & \cdots & y_{N, T}
\end{array}\right], \boldsymbol{\Phi}=\left[\begin{array}{ccc}
\phi_{1,1} & \cdots & \phi_{G, 1} \\
\vdots & \ddots & \vdots \\
\phi_{1, N} & \cdots & \phi_{G, N}
\end{array}\right], \boldsymbol{\Theta}=\left[\begin{array}{ccc}
\theta_{1,1} & \cdots & \theta_{H, 1} \\
\vdots & \ddots & \vdots \\
\theta_{1, N} & \cdots & \theta_{H, N}
\end{array}\right],
$$

while ordinary difference operator matrix $\boldsymbol{\Delta}^{d}=\prod_{i=1}^{d}\left(\left[\begin{array}{ll}\mathbf{0} & \boldsymbol{I}_{T-i}\end{array}\right]-\left[\begin{array}{ll}\boldsymbol{I}_{T-i} & \mathbf{0}\end{array}\right]\right)$. The unique global minimum $\overline{\boldsymbol{Y}}_{\mid T}$ of objective function (2) exists and satisfies:

$$
\operatorname{Vec}\left(\overline{\boldsymbol{Y}}_{T}\right)=\left[\boldsymbol{I}_{N T}+\left(\boldsymbol{I}_{N} \otimes\left(\lambda^{2}\left(\boldsymbol{\Delta}^{d}\right)^{\top}\left(\boldsymbol{\Delta}^{d}\right)\right)\right)+\left(\left(\gamma^{2} \boldsymbol{\Phi} \boldsymbol{\Phi}^{\top}+\psi^{2} \boldsymbol{\Theta} \boldsymbol{\Theta}^{\top}\right) \otimes \boldsymbol{I}_{T}\right)\right]^{-1}\left[\boldsymbol{I}_{N T}+\left(\left(\gamma^{2} \boldsymbol{\Phi} \boldsymbol{\Phi}^{\top}\right) \otimes \boldsymbol{I}_{T}\right)\right] \operatorname{Vec}(\boldsymbol{Y})
$$

Proof. See Appendix A.

\section{B. The Generalized Multivariate Linear Filter}

We define the Generalized Multivariate Linear Filter as that trend component estimator $\left\{\left\{\bar{y}_{i, t \mid T}\right\}_{i=1}^{N}\right\}_{t=1}^{T}$ which minimizes objective function:

$$
\begin{aligned}
& S\left(\left\{\left\{\bar{y}_{i, t}\right\}_{i=1}^{N}\right\}_{t=1}^{T}\right)=\sum_{i=1}^{N} \sum_{t=1}^{T} \hat{y}_{i, t}^{2}+\sum_{i=1}^{N} \sum_{d=1}^{D} \lambda_{d, i}^{2} \sum_{t=d+1}^{T}\left(\Delta^{d} \bar{y}_{i, t}\right)^{2} \\
& +\sum_{g=1}^{G} \gamma_{g}^{2} \sum_{t=P_{1}+1}^{T-P_{2}}\left(\sum_{i=1}^{N} \sum_{p=-P_{1}}^{P_{2}} \phi_{g, i, p} \hat{y}_{i, t+p}\right)^{2}+\sum_{h=1}^{H} \psi_{h}^{2} \sum_{t=Q_{1}+1}^{T-Q_{2}}\left(\sum_{i=1}^{N} \sum_{q=-Q_{1}}^{Q_{2}} \theta_{h, i, q} \bar{y}_{i, t+q}\right)^{2} .
\end{aligned}
$$

This minimization problem strikes a balance between minimizing the sum of squares of the cyclical components and the sum of squares of the ordinary difference of variable specific order $d_{i}$ of the trend components, where $\lambda_{d, i}=0$ for all $d \neq d_{i}$. As $\lambda_{d, i}$ increases, the estimated trend component becomes smoother, converging to a deterministic polynomial of degree $d_{i}-1$ in the limit as $\lambda_{d, i} \rightarrow \infty$. We therefore recommend choosing the minimum value of $d_{i}$ for which $\left\{\Delta^{d_{i}-1} y_{i, t}\right\}_{t=d_{i}}^{T}$ does not exhibit a long run trend for all $i=1, \ldots, N$. This minimization problem also quadratically penalizes deviations of $G$ linearly independent dynamic linear combinations of up to lag order $P_{1}$ and lead order $P_{2}$ of the cyclical components from zero, and $H$ linearly independent dynamic linear combinations of up to lag order $Q_{1}$ and lead order $Q_{2}$ of the trend 
components from zero, where $G \leq N$ and $H \leq N$. As $\gamma_{g}$ or $\psi_{h}$ increases, the estimated cyclical or trend components more closely satisfy the stochastic linear restriction imposed on their comovement, which becomes deterministic in the limit as $\gamma_{g} \rightarrow \infty$ or $\psi_{h} \rightarrow \infty$, respectively. This minimization problem reduces to that associated with our OMLF for: i) $d_{i}=d$ and $\lambda_{d, i}=\lambda_{d}$ for all $i=1, \ldots, N$; ii) $P_{1}=P_{2}=0$ and $\gamma_{g}=\gamma$ for all $g=1, \ldots, G$; and iii) $Q_{1}=Q_{2}=0$ and $\psi_{h}=\psi$ for all $h=1, \ldots, H$.

Proposition 2. Let $\overline{\boldsymbol{Y}}_{T}$ denote the Generalized Multivariate Linear Filter. Using matrix notation, objective function (4) may be expressed as

$$
\begin{aligned}
S(\overline{\boldsymbol{Y}})= & \operatorname{Tr}\left(\hat{\boldsymbol{Y}}^{\top} \hat{\boldsymbol{Y}}\right)+\sum_{d=1}^{D} \operatorname{Tr}\left(\left(\boldsymbol{\Delta}^{d} \overline{\boldsymbol{Y}} \boldsymbol{\Lambda}_{d}\right)^{\top}\left(\boldsymbol{\Delta}^{d} \overline{\boldsymbol{Y}} \boldsymbol{\Lambda}_{d}\right)\right) \\
& +\operatorname{Tr}\left(\left(\sum_{p=-P_{1}}^{P_{2}} \boldsymbol{L}_{\boldsymbol{P}}^{p} \hat{\boldsymbol{Y}} \boldsymbol{\Phi}_{p} \boldsymbol{\Gamma}\right)^{\top}\left(\sum_{p=-P_{1}}^{P_{2}} \boldsymbol{L}_{\boldsymbol{P}}^{p} \hat{\boldsymbol{Y}} \boldsymbol{\Phi}_{p} \boldsymbol{\Gamma}\right)\right)+\operatorname{Tr}\left(\left(\sum_{q=-Q_{1}}^{Q_{2}} \boldsymbol{L}_{\boldsymbol{Q}}^{q} \overline{\boldsymbol{Y}} \boldsymbol{\Theta}_{q} \boldsymbol{\Psi}\right)^{\top}\left(\sum_{q=-Q_{1}}^{Q_{2}} \boldsymbol{L}_{\boldsymbol{Q}}^{q} \overline{\boldsymbol{Y}} \boldsymbol{\Theta}_{q} \boldsymbol{\Psi}\right)\right),
\end{aligned}
$$

where

$$
\begin{gathered}
\boldsymbol{Y}=\left[\begin{array}{ccc}
y_{1,1} & \cdots & y_{N, 1} \\
\vdots & \ddots & \vdots \\
y_{1, T} & \cdots & y_{N, T}
\end{array}\right], \boldsymbol{\Lambda}_{d}=\left[\begin{array}{ccc}
\lambda_{d, 1} & \cdots & 0 \\
\vdots & \ddots & \vdots \\
0 & \cdots & \lambda_{d, N}
\end{array}\right], \boldsymbol{\Phi}_{p}=\left[\begin{array}{ccc}
\phi_{1,1, p} & \cdots & \phi_{G, 1, p} \\
\vdots & \ddots & \vdots \\
\phi_{1, N, p} & \cdots & \phi_{G, N, p}
\end{array}\right], \boldsymbol{\Gamma}=\left[\begin{array}{ccc}
\gamma_{1} & \cdots & 0 \\
\vdots & \ddots & \vdots \\
0 & \cdots & \gamma_{G}
\end{array}\right], \\
\boldsymbol{\Theta}_{q}=\left[\begin{array}{ccc}
\theta_{1,1, q} & \cdots & \theta_{H, 1, q} \\
\vdots & \ddots & \vdots \\
\theta_{1, N, q} & \cdots & \theta_{H, N, q}
\end{array}\right], \boldsymbol{\Psi}=\left[\begin{array}{ccc}
\psi_{1} & \cdots & 0 \\
\vdots & \ddots & \vdots \\
0 & \cdots & \psi_{H}
\end{array}\right],
\end{gathered}
$$

while ordinary difference operator matrix $\boldsymbol{\Delta}^{d}=\prod_{i=1}^{d}\left(\left[\begin{array}{ll}\mathbf{0} & \boldsymbol{I}_{T-i}\end{array}\right]-\left[\begin{array}{ll}\boldsymbol{I}_{T-i} & \mathbf{0}\end{array}\right]\right)$, and lag operator matrices $\boldsymbol{L}_{\boldsymbol{P}}^{p}=\left[\begin{array}{ll}\boldsymbol{I}_{T-P_{1}-P_{2}} & \mathbf{0}\end{array}\right]\left[\begin{array}{ll}\mathbf{0} & \boldsymbol{I}_{T-P_{1}}\end{array}\right]\left[\begin{array}{ll}\boldsymbol{I}_{T-p} & \mathbf{0}\end{array}\right]^{\mathbf{i f}} p \leq 0 \quad$ and $\boldsymbol{L}_{\boldsymbol{P}}^{p}=\left[\begin{array}{ll}\mathbf{0} & \boldsymbol{I}_{T-P_{1}-P_{2}}\end{array}\right]$ $\left[\begin{array}{ll}\boldsymbol{I}_{T-P_{2}} & \mathbf{0}\end{array}\right]\left[\begin{array}{ll}\mathbf{0} & \boldsymbol{I}_{T-p}\end{array}\right]$ otherwise, and $\boldsymbol{L}_{\boldsymbol{Q}}^{q}=\left[\begin{array}{ll}\boldsymbol{I}_{T-Q_{1}-Q_{2}} & \mathbf{0}\end{array}\right]\left[\begin{array}{ll}\mathbf{0} & \boldsymbol{I}_{T-Q_{1}}\end{array}\right]\left[\begin{array}{ll}\boldsymbol{I}_{T-q} & \mathbf{0}\end{array}\right]$ if $q \leq 0$ and $\boldsymbol{L}_{\boldsymbol{Q}}^{q}=\left[\begin{array}{cc}\mathbf{0} & \boldsymbol{I}_{T-Q_{1}-Q_{2}}\end{array}\right]\left[\begin{array}{ll}\boldsymbol{I}_{T-Q_{2}} & \mathbf{0}\end{array}\right]\left[\begin{array}{ll}\mathbf{0} & \boldsymbol{I}_{T-q}\end{array}\right]$ otherwise. The unique global minimum $\overline{\boldsymbol{Y}}_{\mid T}$ of objective function (5) exists and satisfies:

$$
\begin{gathered}
\operatorname{Vec}\left(\overline{\boldsymbol{Y}}_{\boldsymbol{T}}\right)=\left[\boldsymbol{I}_{N T}+\sum_{d=1}^{D}\left(\left(\boldsymbol{\Lambda}_{d} \boldsymbol{\Lambda}_{d}^{\top}\right) \otimes\left(\left(\boldsymbol{\Delta}^{d}\right)^{\top}\left(\boldsymbol{\Delta}^{d}\right)\right)\right)+\sum_{p=-P_{p}}^{P_{2}}\left(\left(\left(\boldsymbol{\Phi}_{p} \boldsymbol{\Gamma}\right)\left(\boldsymbol{\Phi}_{p} \boldsymbol{\Gamma}\right)^{\top}\right) \otimes\left(\left(\boldsymbol{L}_{P}^{p}\right)^{\top}\left(\boldsymbol{L}_{P}^{p}\right)\right)\right)+\sum_{q=-Q_{1}}^{Q_{2}}\left(\left(\left(\boldsymbol{\Theta}_{q} \boldsymbol{\Psi}\right)\left(\boldsymbol{\Theta}_{q} \boldsymbol{\Psi}\right)^{\top}\right) \otimes\left(\left(\boldsymbol{L}_{Q}^{q}\right)^{\top}\left(\boldsymbol{L}_{Q}^{q}\right)\right)\right)\right]^{-1} \\
\cdot\left[\boldsymbol{I}_{N T}+\sum_{p=-P_{1}}^{P_{2}}\left(\left(\left(\boldsymbol{\Phi}_{p} \boldsymbol{\Gamma}\right)\left(\boldsymbol{\Phi}_{p} \boldsymbol{\Gamma}\right)^{\top}\right) \otimes\left(\left(\boldsymbol{L}_{P}^{p}\right)^{\top}\left(\boldsymbol{L}_{P}^{p}\right)\right)\right)\right] \operatorname{Vec}(\boldsymbol{Y}) .
\end{gathered}
$$

Proof. See Appendix B.

\section{The New Keynesian Model}

We consider an extension of the basic New Keynesian model of a closed economy documented in Woodford (2003) and Galí (2015). This economy consists of households and firms that optimize intertemporally, interacting with the government in an uncertain environment to determine equilibrium prices and quantities under rational expectations in output, labor and financial markets. 


\section{A. Households}

The representative infinitely lived household indexed by $i \in[0,1]$ has preferences defined over consumption $C_{i, s}$ and labor supply $L_{i, s}$ represented by intertemporal utility function

$$
U_{i, t}=\mathrm{E}_{t} \sum_{s=t}^{\infty} \beta^{s-t} u\left(C_{i, s}, L_{i, s}\right),
$$

where $\mathrm{E}_{t}$ denotes the expectations operator conditional on information available in period $t$, and $0<\beta<1$. The intratemporal utility function is additively separable and represents external habit formation preferences in consumption,

$$
u\left(C_{i, s}, L_{i, s}\right)=\frac{\left(C_{i, s}-\alpha C_{s-1}\right)^{1-1 / \sigma}}{1-1 / \sigma}-C_{s} \frac{\left(C_{s}-\alpha C_{s-1}\right)^{-1 / \sigma}}{1+1 / \eta}\left(\frac{1-\chi}{1-u_{s}}\right)^{1 / \eta}\left(\frac{L_{i, s}}{N_{s}}\right)^{1+1 / \eta},
$$

where $0 \leq \alpha<1, \sigma>0,0<\chi<1$ and $\eta>0$. The unemployment rate $u_{s}$ measures the share of the labor force $N_{s}$ in unemployment $U_{s}$, that is $u_{s}=U_{s} / N_{s}$, where unemployment equals the labor force less employment $L_{s}$, that is $U_{s}=N_{s}-L_{s}$.

The representative household enters period $s$ in possession of previously purchased nominal bonds $B_{i, s}$ which yield interest at risk free rate $i_{s-1}$, and holds a diversified portfolio of shares $\left\{S_{i, j, s}\right\}_{j=0}^{1}$ in intermediate good firms which pay dividends $\left\{\Pi_{j, s}\right\}_{j=0}^{1}$. During period $s$, it supplies labor service $L_{i, s}$, earning labor income at nominal wage $W_{s}$. These sources of wealth are summed in household dynamic budget constraint:

$$
B_{i, s+1}+\int_{0}^{1} V_{j, s} S_{i, j, s+1} d j=\left(1+i_{s-1}\right) B_{i, s}+\int_{0}^{1}\left(\Pi_{j, s}+V_{j, s}\right) S_{i, j, s} d j+W_{s} L_{i, s}-P_{s} C_{i, s} .
$$

According to this dynamic budget constraint, at the end of period $s$, the representative household purchases bonds $B_{i, s+1}$, and a diversified portfolio of shares $\left\{S_{i, j, s+1}\right\}_{j=0}^{1}$ at prices $\left\{V_{j, s}\right\}_{j=0}^{1}$. Finally, it purchases final consumption good $C_{i, s}$ at price $P_{s}$.

In period $t$, the representative household chooses state contingent sequences for consumption $\left\{C_{i, s}\right\}_{s=t}^{\infty}$, labor supply $\left\{L_{i, s}\right\}_{s=t}^{\infty}$, bond holdings $\left\{B_{i, s+1}\right\}_{s=t}^{\infty}$ and share holdings $\left\{\left\{S_{i, j, s+1}\right\}_{j=0}^{1}\right\}_{s=t}^{\infty}$ to maximize intertemporal utility function (7) subject to dynamic budget constraint (9), and terminal nonnegativity constraints $B_{i, T+1} \geq 0$ and $S_{i, j, T+1} \geq 0$ for $T \rightarrow \infty$. This utility maximization problem yields necessary first order conditions

$$
\begin{aligned}
& u_{C}\left(C_{i, t}, L_{i, t}\right)=P_{t} \lambda_{i, t}, \\
& -u_{L}\left(C_{i, t}, L_{i, t}\right)=W_{t} \lambda_{i, t}, \\
& \lambda_{i, t}=\beta\left(1+i_{t}\right) \mathrm{E}_{t} \lambda_{i, t+1}, \\
& V_{j, t} \lambda_{i, t}=\beta \mathrm{E}_{t}\left(\Pi_{j, t+1}+V_{j, t+1}\right) \lambda_{i, t+1},
\end{aligned}
$$


where $\lambda_{i, s}$ denotes the Lagrange multiplier associated with the period $s$ household dynamic budget constraint.

In equilibrium, combination of necessary first order conditions (10) and (12) yields intertemporal optimality condition

$$
\mathrm{E}_{t} \frac{\beta u_{C}\left(C_{t+1}, L_{t+1}\right)}{u_{C}\left(C_{t}, L_{t}\right)} \frac{P_{t}}{P_{t+1}}\left(1+i_{t}\right)=1,
$$

which equates the expected present value of the gross real interest rate to one. Finally, combination of necessary first order conditions (10) and (11) yields intratemporal optimality condition

$$
-\frac{u_{L}\left(C_{t}, L_{t}\right)}{u_{C}\left(C_{t}, L_{t}\right)}=\frac{W_{t}}{P_{t}}
$$

which equates the marginal rate of substitution between leisure and consumption to the real wage.

\section{B. Firms}

The representative intermediate good firm indexed by $j \in[0,1]$ sells shares $\left\{S_{i, j, t+1}\right\}_{i=0}^{1}$ to households at price $V_{j, t}$. Recursive forward substitution for $V_{j, t+s}$ with $s>0$ in necessary first order condition (13) reveals that the pre-dividend stock market value of the representative intermediate good firm equals the expected present value of current and future dividend payments:

$$
\Pi_{j, t}+V_{j, t}=\mathrm{E}_{t} \sum_{s=t}^{\infty} \frac{\beta^{s-t} \lambda_{s}}{\lambda_{t}} \Pi_{j, s} .
$$

Shares entitle households to dividend payments equal to profits $\Pi_{j, s}$, defined as revenues from sales of differentiated intermediate output good $Y_{j, s}$ at price $P_{j, s}$ less expenditures on labor service $L_{j, s}$ :

$$
\Pi_{j, s}=P_{j, s} Y_{j, s}-W_{s} L_{j, s} .
$$

The representative intermediate good firm rents labor service $L_{j, s}$ given productivity coefficient $A_{s}$ to produce differentiated intermediate output good $Y_{j, s}$ according to production function

$$
Y_{j, s}=A_{s}\left(L_{j, s}\right)^{\phi},
$$

where $A_{s}>0$. This production function is homogeneous of degree $\phi$, where $\phi>0$.

In period $t$, the representative intermediate good firm chooses a state contingent sequence for employment $\left\{L_{j, s}\right\}_{s=t}^{\infty}$ to maximize pre-dividend stock market value (16) subject to production function (18). This value maximization problem yields necessary first order condition

$$
\Phi_{j, t}=\frac{1}{\phi} \frac{W_{t} L_{j, t}}{P_{t} Y_{j, t}},
$$


where $P_{s} \Phi_{j, s}$ denotes the Lagrange multiplier associated with the period $s$ production technology constraint. This necessary first order condition equates real marginal cost $\Phi_{j, t}$ to the ratio of the real wage to the marginal product of labor.

There exist a large number of perfectly competitive firms which combine differentiated intermediate output goods $Y_{j, t}$ supplied by intermediate good firms to produce final output good $Y_{t}$ according to production function

$$
Y_{t}=\left[\int_{0}^{1}\left(Y_{j, t}\right)^{\frac{\theta-1}{\theta}} d j\right]^{\frac{\theta}{\theta-1}},
$$

where $\theta>1$. The representative final good firm maximizes profits derived from production of the final output good with respect to inputs of intermediate output goods, implying demand functions:

$$
Y_{j, t}=\left(\frac{P_{j, t}}{P_{t}}\right)^{-\theta} Y_{t} .
$$

Since the production function exhibits constant returns to scale, in equilibrium the representative final good firm earns zero profit, implying aggregate price index:

$$
P_{t}=\left[\int_{0}^{1}\left(P_{j, t}\right)^{1-\theta} d j\right]^{\frac{1}{1-\theta}} .
$$

Clearing of the final output good market requires that production of the final output good equal the total demand of households, that is $Y_{t}=C_{t}$.

In an extension of the model of nominal price rigidity proposed by Calvo (1983) following Smets and Wouters (2003), each period a randomly selected fraction $1-\omega$ of intermediate good firms adjust their price optimally, where $0 \leq \omega<1$. The remaining fraction $\omega$ of intermediate good firms adjust their price to account for past inflation according to partial indexation rule

$$
P_{j, t}=\left(\frac{P_{t-1}}{P_{t-2}}\right)^{\gamma}\left(\frac{\bar{P}_{t-1}}{\bar{P}_{t-2}}\right)^{1-\gamma} P_{j, t-1},
$$

where $0 \leq \gamma \leq 1$. If the representative intermediate good firm can adjust its price optimally in period $t$, then it does so to maximize pre-dividend stock market value (16) subject to production function (18), intermediate output good demand function (21), and the assumed form of nominal price rigidity. We consider a symmetric equilibrium under which all intermediate good firms that adjust their price optimally in period $t$ choose a common price $P_{t}^{*}$ given by necessary first order condition: 


$$
\frac{P_{t}^{*}}{P_{t}}=\frac{\theta}{\theta-1} \frac{\mathrm{E}_{t} \sum_{s=t}^{\infty}(\omega)^{s-t} \frac{\beta^{s-t} \lambda_{s}}{\lambda_{t}} \Phi_{j, s}\left[\left(\frac{P_{t-1}}{P_{s-1}}\right)^{\gamma}\left(\frac{\bar{P}_{t-1}}{\bar{P}_{s-1}}\right)^{1-\gamma} \frac{P_{s}}{P_{t}}\right]^{\theta} P_{s} Y_{s}}{\mathrm{E}_{t} \sum_{s=t}^{\infty}(\omega)^{s-t} \frac{\beta^{s-t} \lambda_{s}}{\lambda_{t}}\left[\left(\frac{P_{t-1}}{P_{s-1}}\right)^{\gamma}\left(\frac{\bar{P}_{t-1}}{\bar{P}_{s-1}}\right)^{1-\gamma} \frac{P_{s}}{P_{t}}\right]^{\theta-1} P_{s} Y_{s}} .
$$

This necessary first order condition equates the expected present value of marginal revenue to the expected present value of marginal cost. Aggregate price index (22) equals an average of the price set by the fraction $1-\omega$ of intermediate good firms that adjust their price optimally in period $t$, and the average of the prices set by the remaining fraction $\omega$ of intermediate good firms that adjust their price according to indexation rule (23):

$$
P_{t}=\left[(1-\omega)\left(P_{t}^{*}\right)^{1-\theta}+\omega\left[\left(\frac{P_{t-1}}{P_{t-2}}\right)^{\gamma}\left(\frac{\bar{P}_{t-1}}{\bar{P}_{t-2}}\right)^{1-\gamma} P_{t-1}\right]^{1-\theta}\right]^{\frac{1}{1-\theta}} .
$$

Since those intermediate good firms able to adjust their price optimally in period $t$ are selected randomly from among all intermediate good firms, the average price set by the remaining intermediate good firms equals the value of the aggregate price index that prevailed during period $t-1$, rescaled to account for past inflation.

\section{Policy}

The government consists of a monetary authority which implements monetary policy through control of the nominal policy interest rate according to a monetary policy rule exhibiting partial adjustment dynamics of the form

$$
i_{t}-\tilde{i}_{t}=\rho\left(i_{t-1}-\tilde{i}_{t-1}\right)+(1-\rho)\left[\xi\left(\pi_{t}-\pi\right)-\phi \zeta\left(u_{t}-\chi\right)\right],
$$

where $0 \leq \rho<1, \xi>1$ and $\zeta>0$. As specified, the deviation of the nominal policy interest rate from its flexible price equilibrium value depends on a weighted average of its past deviation and its desired deviation, which in turn is increasing in the contemporaneous deviation of inflation from its target value, and is decreasing in the contemporaneous deviation of the unemployment rate from its flexible price equilibrium value. In flexible price equilibrium, this monetary policy rule reduces to $\tilde{\pi}_{t}=\pi$.

\section{Equilibrium}

A rational expectations equilibrium in this New Keynesian model of a closed economy consists of state contingent sequences of allocations for households and firms that solve their constrained optimization problems given prices and policy, together with a state contingent sequence of instrument settings for the government that satisfies its policy rule given prices, with supporting prices such that all markets clear. 
Let $\hat{x}_{t}$ denote the deviation of variable $x_{t}$ from its steady state equilibrium value $\bar{x}_{t}$. Analytically linearizing the equilibrium conditions of our New Keynesian model around a stationary deterministic steady state equilibrium, and consolidating them by substituting out intermediate variables, yields

$$
\begin{aligned}
& \hat{\pi}_{t}=\frac{\gamma}{1+\gamma \beta} \hat{\pi}_{t-1}+\frac{\beta}{1+\gamma \beta} \mathrm{E}_{t} \hat{\pi}_{t+1}-\frac{(1-\omega)(1-\omega \beta)}{\omega(1+\gamma \beta)} \hat{u}_{t}, \\
& \hat{u}_{t}=\frac{\alpha}{1+\alpha} \hat{u}_{t-1}+\frac{1}{1+\alpha} \mathrm{E}_{t} \hat{u}_{t+1}+\frac{\sigma}{\phi} \frac{1-\alpha}{1+\alpha}\left[\hat{r}_{t}-\frac{1}{\sigma} \mathrm{E}_{t} \Delta \frac{\left(\hat{a}_{t+1}+\phi \hat{n}_{t+1}\right)-\alpha\left(\hat{a}_{t}+\phi \hat{n}_{t}\right)}{1-\alpha}\right], \\
& \hat{u}_{t}=-\frac{1}{\phi}\left[\hat{y}_{t}-\left(\hat{a}_{t}+\phi \hat{n}_{t}\right)\right], \\
& \hat{i}_{t}-\hat{\tilde{i}}=\rho\left(\hat{i}_{t-1}-\hat{\tilde{i}}_{t-1}\right)+(1-\rho)\left(\xi \hat{\pi}_{t}-\phi \zeta \hat{u}_{t}\right),
\end{aligned}
$$

where the real interest rate $\hat{r}_{t}$ is defined as $\hat{r}_{t}=\hat{i}_{t}-\mathrm{E}_{t} \hat{\pi}_{t+1}$, while the inflation rate $\hat{\pi}_{t}$ is defined as $\hat{\pi}_{t}=\hat{p}_{t}-\hat{p}_{t-1}$, and lowercase variables denote the natural logarithms of their uppercase counterparts. This steady state equilibrium features zero inflation, productivity growth, and labor force growth.

Let $\hat{\hat{x}}_{t}$ denote the deviation of variable $x_{t}$ from its flexible price equilibrium value $\tilde{x}_{t}$, otherwise referred to as its natural or potential value. Our linearized New Keynesian model may be restated as

$$
\begin{aligned}
& \hat{\hat{\pi}}_{t}=\frac{\gamma}{1+\gamma \beta} \hat{\hat{\pi}}_{t-1}+\frac{\beta}{1+\gamma \beta} \mathrm{E}_{t} \hat{\hat{\pi}}_{t+1}+\frac{(1-\omega)(1-\omega \beta)}{\omega(1+\gamma \beta)} \frac{1}{\phi} \hat{\hat{y}}_{t}, \\
& \hat{\hat{y}}_{t}=\frac{\alpha}{1+\alpha} \hat{\hat{y}}_{t-1}+\frac{1}{1+\alpha} \mathrm{E}_{t} \hat{\hat{y}}_{t+1}-\sigma \frac{1-\alpha}{1+\alpha} \hat{\hat{r}}_{t}, \\
& \hat{\hat{u}}_{t}=-\frac{1}{\phi} \hat{\hat{y}}_{t}, \\
& \hat{\hat{i}}_{t}=\rho \hat{\hat{i}}_{t-1}+(1-\rho)\left(\xi \hat{\hat{\pi}}_{t}+\zeta \hat{\hat{y}}_{t}\right),
\end{aligned}
$$

where the real interest rate gap $\hat{\hat{r}}_{t}$ satisfies $\hat{\hat{r}}_{t}=\hat{\hat{i}}_{t}-\mathrm{E}_{t} \hat{\hat{\pi}}_{t+1}$, while the real natural rate of interest satisfies $\hat{\tilde{r}}_{t}=\frac{1}{\sigma} \mathrm{E}_{t} \Delta \frac{\tilde{\tilde{y}}_{t+1}-\alpha \hat{y}_{t}}{1-\alpha}$, and potential output satisfies $\hat{\tilde{y}}_{t}=\hat{a}_{t}+\phi \hat{n}_{t}$. This model consists of a Phillips curve dynamically relating the inflation gap to the output gap, an Euler equation dynamically relating the output gap to the real interest rate gap, a version of Okun's law statically relating the unemployment rate gap to the output gap, and a Taylor rule dynamically relating the nominal policy interest rate gap to the inflation and output gaps. 


\section{Estimation of Potential OUtPut and Natural Rates}

The parameters and unobserved components of our New Keynesian model could be jointly estimated within a linear unobserved components framework by full information maximum likelihood. This would entail augmenting its approximate multivariate linear rational expectations representation with structural shocks, solving for its reduced form, casting it in linear state space form, evaluating the conditional loglikelihood function using the filter due to Kalman (1960), and numerically maximizing it with respect to the structural parameters and innovation variances. Alternatively, the structural shocks could be incorporated into the microeconomic foundations of the model, the structural parameters could be estimated conditional on priors concerning their values, or the unobserved components could be estimated conditional on judgment regarding their paths. Finally, given terminal conditions obtained from the final recursive forward evaluation of the filter due to Kalman (1960), which yields one-sided unobserved components estimates, twosided estimates could be generated through recursive backward evaluation of the computationally efficient smoother associated with de Jong (1989).

Full information maximum likelihood estimation of linear unobserved components models has well defined optimality properties. As discussed in Harvey (1993) and Hamilton (1994), if the model is correctly specified and its parameters are identified, then the parameter estimates are consistent and asymptotically normal, while if in addition its innovations and initial conditions are normally distributed, then the unobserved components estimates are conditionally normally distributed and have minimum mean squared error. However, all macroeconomic models are misspecified, and the parameters of linear unobserved components models are generally only locally identified. While conditioning on well specified priors when estimating parameters or judgment when estimating unobserved components can yield mean squared error reductions by mitigating model misspecification and identification problems, formulating such priors or judgment can be difficult. Finally, full information maximum likelihood estimation of linear unobserved components models can be computationally complex, yielding unobserved components estimates that can be sensitive to initial conditions.

\section{A. Restrictions}

To mitigate model misspecification and identification problems when jointly estimating potential output, the natural rate of unemployment and the natural rate of interest, we instead use our GMLF, conditional on selected approximate linear equilibrium conditions from our New Keynesian model, and calibrated values of a subset of its structural parameters. In particular, to avoid propagating model misspecification problems throughout its reduced form, we condition only on those approximate linear equilibrium conditions that are relatively well specified, and evaluate them under perfect foresight:

$$
\hat{\hat{\pi}}_{t}=\frac{\gamma}{1+\gamma \beta} \hat{\hat{\pi}}_{t-1}+\frac{\beta}{1+\gamma \beta} \hat{\hat{\pi}}_{t+1}+\frac{(1-\omega)(1-\omega \beta)}{\omega(1+\gamma \beta)} \frac{1}{\phi} \hat{\hat{y}}_{t},
$$




$$
\begin{aligned}
& \hat{\hat{y}}_{t}=\frac{\alpha}{1+\alpha} \hat{\hat{y}}_{t-1}+\frac{1}{1+\alpha} \hat{\hat{y}}_{t+1}-\sigma \frac{1-\alpha}{1+\alpha}\left(\hat{\hat{i}}_{t}-\hat{\hat{\pi}}_{t+1}\right), \\
& \hat{\hat{u}}_{t}=-\frac{1}{\phi} \hat{\hat{y}}_{t}, \\
& \Delta \tilde{i}_{t}-\Delta \tilde{\pi}_{t+1}=\frac{1}{\sigma} \Delta^{2} \frac{\tilde{y}_{t+1}-\alpha \tilde{y}_{t}}{1-\alpha} .
\end{aligned}
$$

These approximate linear equilibrium conditions are the Phillips curve, the Euler equation, the Okun's law relationship, and a transformation of the natural rate relationship. The Taylor rule is omitted as it is unlikely to provide an empirically adequate description of the conduct of monetary policy over a long sample period spanning multiple operating procedures, while the natural rate relationship is differenced as its derivation abstracts from asset risk premia which drive a wedge between the return on saving and the policy interest rate.

The ordered set of observed endogenous variables under consideration consists of the inflation rate, output, the unemployment rate, and the nominal policy interest rate. The sets of approximate linear equilibrium conditions under consideration restrict dynamic interrelationships among the unobserved components of these $N=4$ observed endogenous variables. We treat the deviations of these observed endogenous variables from their flexible price equilibrium values as cyclical components, and these flexible price equilibrium values as trend components. Accordingly, the coefficient matrices associated with our $G=3$ dynamic cyclical restrictions may be stated as

$$
\Phi_{+1}=\left[\begin{array}{ccc}
-\frac{\beta}{1+\gamma \beta} & -\sigma \frac{1-\alpha}{1+\alpha} & 0 \\
0 & -\frac{1}{1+\alpha} & 0 \\
0 & 0 & 0 \\
0 & 0 & 0
\end{array}\right], \Phi_{0}=\left[\begin{array}{ccc}
1 & 0 & 0 \\
-\frac{(1-\omega)(1-\omega \beta)}{\omega(1+\gamma \beta)} \frac{1}{\phi} & 1 & \frac{1}{\phi} \\
0 & 0 & 1 \\
0 & \sigma \frac{1-\alpha}{1+\alpha} & 0
\end{array}\right], \Phi_{-1}=\left[\begin{array}{ccc}
-\frac{\gamma}{1+\gamma \beta} & 0 & 0 \\
0 & -\frac{\alpha}{1+\alpha} & 0 \\
0 & 0 & 0 \\
0 & 0 & 0
\end{array}\right] \text {, }
$$

where maximum lag order $P_{1}=1$ and lead order $P_{2}=1$. These cyclical restrictions are nonredundant, as they are linearly independent. In parallel, the coefficient matrices associated with our $H=1$ dynamic trend restriction may be stated as

$$
\boldsymbol{\Theta}_{+1}=\left[\begin{array}{c}
-1 \\
-\frac{1}{\sigma} \frac{1}{1-\alpha} \\
0 \\
0
\end{array}\right], \boldsymbol{\Theta}_{0}=\left[\begin{array}{c}
1 \\
\frac{1}{\sigma} \frac{2+\alpha}{1-\alpha} \\
0 \\
1
\end{array}\right], \boldsymbol{\Theta}_{-1}=\left[\begin{array}{c}
0 \\
-\frac{11+2 \alpha}{\sigma} \frac{1-\alpha}{1-\alpha} \\
0 \\
-1
\end{array}\right], \boldsymbol{\Theta}_{-2}=\left[\begin{array}{c}
0 \\
\frac{1}{\sigma} \frac{\alpha}{1-\alpha} \\
0 \\
0
\end{array}\right],
$$

where maximum lag order $Q_{1}=2$ and lead order $Q_{2}=1$. We calibrate the structural parameters that enter into these restrictions to lie within the range of estimates reported in the existing empirical literature. In particular, the subjective discount factor parameter $\beta$ is set to imply an annualized discount rate of 3 percent, while the habit persistence parameter $\alpha$ is set to 0.800 , and the intertemporal elasticity of substitution parameter $\sigma$ is set to 1.000. Furthermore, the production degree of homogeneity parameter $\phi$ is set to imply an Okun's law coefficient of 
-0.500 . Finally, the partial indexation parameter $\gamma$ is set to 0.800 , while the nominal rigidity parameter $\omega$ is set to imply an average reoptimization interval of 6 quarters.

\section{B. Results}

We use our GMLF to jointly estimate potential output, the natural rate of unemployment and the natural rate of interest for the United States, conditional on these approximate linear equilibrium conditions from our calibrated New Keynesian model, which we treat as stochastic restrictions. Measurement of the inflation rate is based on the seasonally adjusted gross domestic product price deflator, of output is based on seasonally adjusted real gross domestic product, of the unemployment rate is based on the seasonally adjusted civilian unemployment rate, and of the nominal policy interest rate is based on the effective federal funds rate expressed as a period average. These time series variables are transformed in line with our New Keynesian model, with the inflation and nominal policy interest rates expressed as quarterly percentage rates, and the natural logarithm of output scaled by a factor of 100 . They span the sample period 1954Q3 through 2018Q2, and were obtained from the FRED database compiled by the Federal Reserve Bank of Saint Louis.

Given this set of time series variables, and the sets of stochastic linear restrictions among their cyclical and trend components under consideration, using our GMLF requires assigning values to the tuning parameters that enter into the objective function that it minimizes. For the inflation, unemployment and nominal policy interest rates - which do not exhibit long run trends - we set the difference orders $d_{i}$ to 1 and the smoothing parameters $\lambda_{1, i}$ to 20 . In contrast, for output which does exhibit a long run trend - we set the difference order $d_{i}$ to 2 and the smoothing parameter $\lambda_{2, i}$ to 400 . For the cyclical restrictions we set the weight parameters $\gamma_{g}$ to 2.000 , thereby quadratically penalizing the deviations of these dynamic linear combinations of the cyclical components from zero somewhat more than the deviations of the cyclical components from zero. Finally, for the trend restriction we set the weight parameter $\psi_{h}$ to 0.002 , as the deviations of this dynamic linear combination of the trend components from zero are much more persistent than those of the dynamic linear combinations of the cyclical components. 
Figure 1. Estimated Trend Components from Multivariate versus Univariate Filters
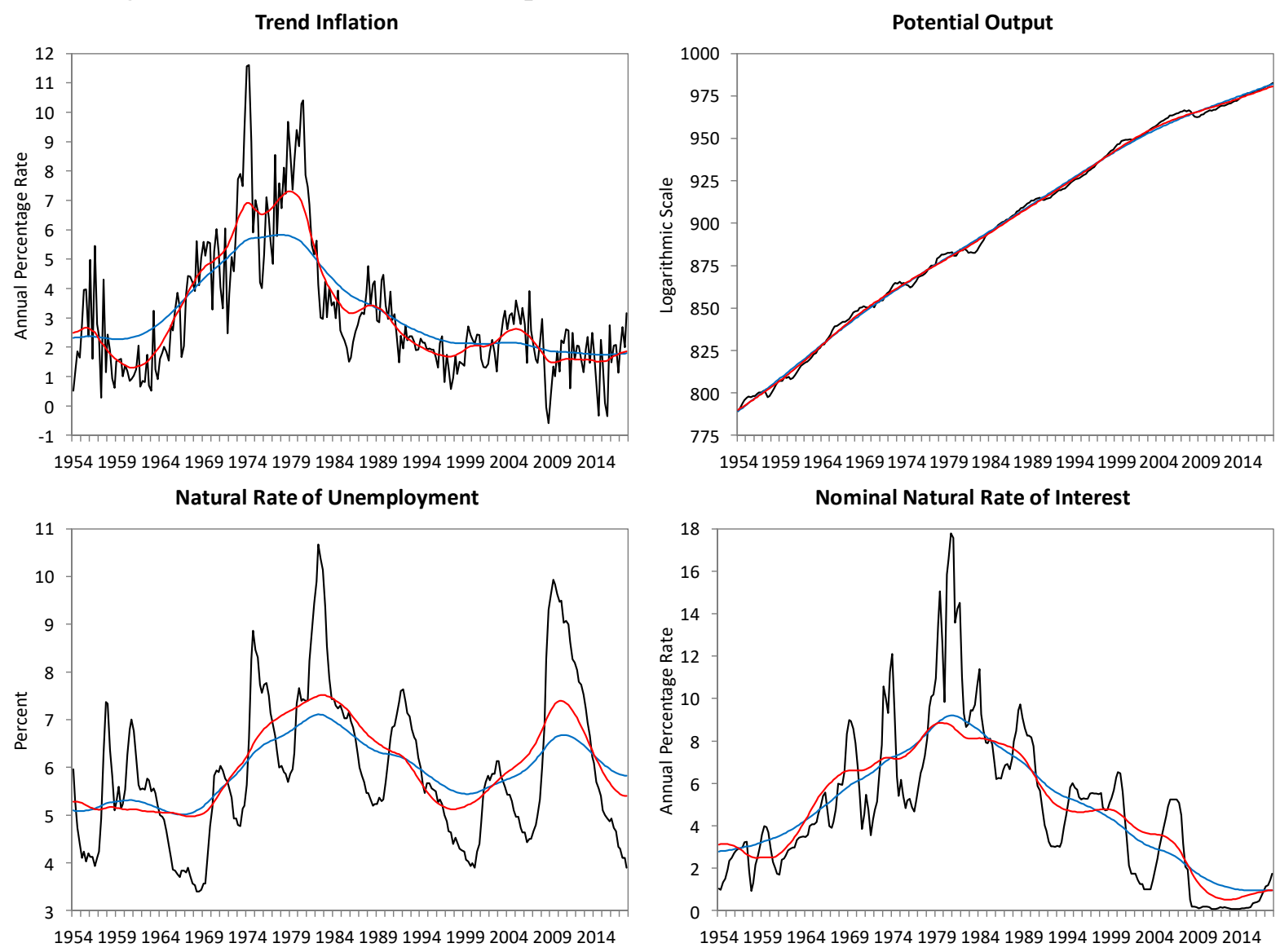

Note: Depicts observed levels $\mathbf{\square}$ versus estimated trend components from multivariate $\boldsymbol{\|}$ and univariate $\boldsymbol{\|}$ filters.

Our multivariate filter based estimates of trend inflation, potential output, the natural rate of unemployment and the natural rate of interest exhibit economically significant and interpretable deviations from those generated using the corresponding univariate filters with matching smoothing parameter values. For trend inflation and the natural rates of unemployment and interest the corresponding univariate filter is that associated with Lucas (1980), while for potential output it is that associated with Hodrick and Prescott (1997). In absolute value, these deviations are up to 1.5 percentage points for trend inflation, 1.4 percent for potential output, 0.7 percentage points for the natural rate of unemployment, and 0.9 percentage points for the natural rate of interest. The cyclical restrictions under consideration pull the multivariate filter based estimates above or below the univariate filter based estimates at cyclical frequencies. For example, the natural rate of unemployment is estimated to have risen more during the Global Financial Crisis when the Okun's law relationship is conditioned on, while the natural rate of interest is estimated to have fallen more in its aftermath when the Euler equation is conditioned on. This reflects the atypically large increase in the unemployment rate gap relative to the decrease in the output gap estimated by the univariate filters during the Global Financial Crisis, and the atypically large fall in the interest rate 
gap relative to the slow increase in the output gap during the subsequent sluggish recovery. ${ }^{2}$ In contrast, the trend restriction under consideration shifts the multivariate filter based estimates above or below the univariate filter based estimates at trend frequencies, influencing them less than the cyclical restrictions given its weight parameter value. Finally, our multivariate filter based output gap estimates track recessions slightly better than the univariate filter based estimates, as measured by the correlation between the direction of change in the output gap and a recession indicator variable.

Figure 2. Estimated Cyclical Components from Multivariate versus Univariate Filters
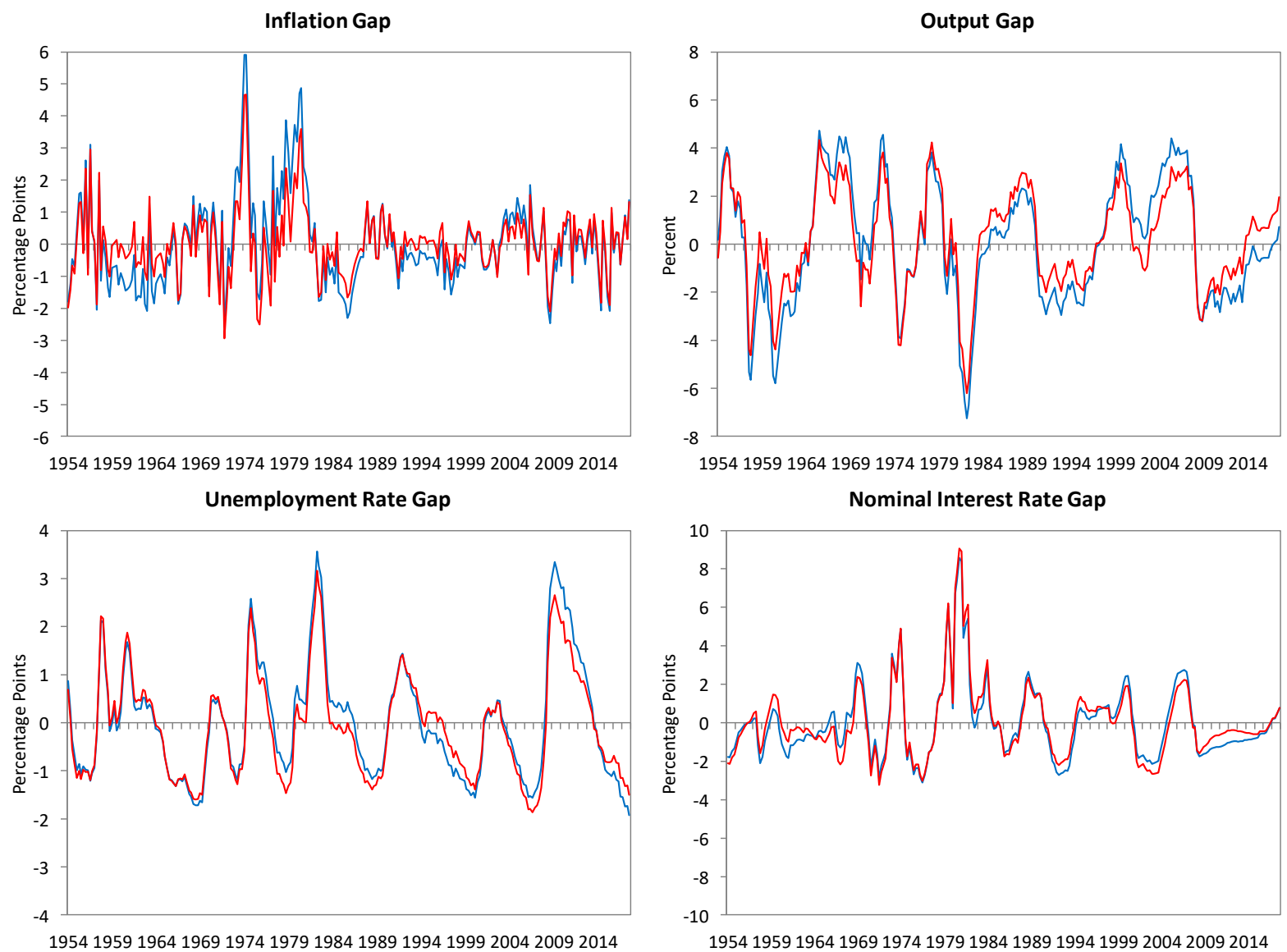

Note: Depicts estimated cyclical components from multivariate $\mathbf{a}$ and univariate $\mathbf{E}$ filters, where shaded regions indicate recessions as dated by the National Bureau of Economic Research.

\section{Robustness}

The dependence of our closed form multivariate linear filters on multiple parameters - entering into both the objective function that they minimize and the stochastic restrictions that they condition on - has advantages and disadvantages. This parametric flexibility provides

\footnotetext{
${ }^{2}$ The interest rate gap only measures the stance of conventional monetary policy, unless an estimated shadow nominal policy interest rate substitutes for the observed nominal policy interest rate when the latter was constrained by the effective lower bound and unconventional monetary policy measures were resorted to.
} 
considerable scope to adjust the relative volatility and theoretical congruence of the cyclical and appropriately differenced trend component estimates. The cost of this flexibility is the potential sensitivity of these estimates to parameter perturbations. Fortunately, sensitivity analysis is computationally simple to conduct.

Table 1. Sensitivity of Estimation Results to Smoothing Parameter Perturbations

\begin{tabular}{|c|c|c|c|c|c|c|c|c|}
\hline & \multicolumn{2}{|c|}{$\overline{\lambda_{1.1}}$} & \multicolumn{2}{|c|}{$\lambda_{2.2}$} & \multicolumn{2}{|c|}{$\bar{~} \overline{\lambda_{1.3}}$} & \multicolumn{2}{|c|}{$\lambda_{1.4}$} \\
\hline & 10 & 40 & 100 & 1,600 & 10 & 40 & 10 & 40 \\
\hline$\hat{\hat{\pi}}_{t}$ & 0.957 & 0.960 & 0.998 & 0.992 & 1.000 & 1.000 & 1.000 & 1.000 \\
\hline$\hat{\hat{y}}_{t}$ & 1.000 & 1.000 & 0.892 & 0.897 & 1.000 & 1.000 & 1.000 & 1.000 \\
\hline$\hat{u}_{t}$ & 1.000 & 1.000 & 0.987 & 0.946 & 0.986 & 0.980 & 1.000 & 1.000 \\
\hline$\hat{i_{t}}$ & 1.000 & 1.000 & 0.983 & 0.895 & 1.000 & 1.000 & 0.886 & 0.932 \\
\hline
\end{tabular}

Note: Reports correlations with the central estimates.

The sensitivity of our multivariate filter based inflation, output, unemployment rate and interest rate gap estimates to parameter perturbations varies widely, measured in terms of correlations with the central estimates. For reference, the correlations between these estimates and those generated using the corresponding univariate filters are 0.918 for the inflation gap, 0.950 for the output gap, 0.972 for the unemployment rate gap, and 0.970 for the interest rate gap. Focusing on material reductions in linear association, sensitivity analysis with respect to the smoothing parameters reveals that adjusting the smoothness of trend inflation only affects the inflation gap estimates, of potential output affects all of the gap estimates, of the natural rate of unemployment only affects the unemployment rate gap estimates, and of the natural rate of interest only affects the interest rate gap estimates. Similarly, sensitivity analysis with respect to the weight parameters reveals that conditioning to varying degrees on the Phillips curve only affects the inflation gap estimates, on the Euler equation affects all of the gap estimates, and on the Okun's law relationship only affects the unemployment rate gap estimates. In contrast, tightening the stochastic restriction representing the natural rate relationship affects none of the gap estimates according to this metric, reflecting the invariance of correlations to constant shifts of variables. In parallel, sensitivity analysis with respect to the structural parameters reveals that the calibration of those parameters that enter only into the Phillips curve, namely the subjective discount factor, partial indexation and nominal rigidity parameters, affects none of the gap estimates, indicative of weak identification. Furthermore, the calibration of those parameters that enter into the Euler equation and natural rate relationship, namely the habit persistence and intertemporal elasticity of substitution parameters, affects all and some of the gap estimates, respectively. In particular, the output and unemployment rate gap estimates are invariant to adjustments to the intertemporal elasticity of substitution parameter, and only depend on upward adjustments to the habit persistence parameter. Finally, the calibration of that parameter that enters into the Okun's law relationship, namely the production degree of homogeneity parameter, only affects the unemployment rate gap estimates. 
Table 2. Sensitivity of Estimation Results to Weight Parameter Perturbations

\begin{tabular}{ccccccccc}
\hline \hline & & \multicolumn{3}{c}{$\gamma_{1}$} & & \multicolumn{2}{c}{$\gamma_{3}$} & \multicolumn{2}{c}{$\psi_{1}$} \\
& 0.000 & 4.000 & 0.000 & 4.000 & 0.000 & 4.000 & 0.000 & 0.004 \\
\hline$\hat{\pi}_{t}$ & 0.928 & 0.974 & 0.993 & 0.979 & 1.000 & 1.000 & 1.000 & 1.000 \\
$\hat{\hat{y}}_{t}$ & 1.000 & 1.000 & 0.960 & 0.973 & 1.000 & 1.000 & 1.000 & 1.000 \\
$\hat{\hat{u}}_{t}$ & 1.000 & 1.000 & 0.982 & 0.995 & 0.972 & 0.990 & 1.000 & 1.000 \\
$\hat{i}_{t}$ & 1.000 & 1.000 & 0.970 & 0.952 & 1.000 & 1.000 & 1.000 & 1.000 \\
\hline \hline
\end{tabular}

Note: Reports correlations with the central estimates.

Table 3. Sensitivity of Estimation Results to Structural Parameter Perturbations

\begin{tabular}{|c|c|c|c|c|c|c|c|c|c|c|c|c|}
\hline & \multicolumn{2}{|c|}{$\bar{\beta}$} & \multicolumn{2}{|c|}{$\alpha$} & \multicolumn{2}{|c|}{$\sigma$} & \multicolumn{2}{|c|}{$\phi$} & \multicolumn{2}{|c|}{$\gamma$} & \multicolumn{2}{|c|}{$\omega$} \\
\hline & $1 / 1.005$ & $1 / 1.010$ & 0.650 & 0.950 & 0.500 & 1.500 & $1 / 0.250$ & $1 / 0.750$ & 0.650 & 0.950 & $(4-1) / 4$ & $(8-1) / 8$ \\
\hline$\hat{\pi}_{t}$ & 1.000 & 1.000 & 0.992 & 0.996 & 0.998 & 0.998 & 1.000 & 1.000 & 1.000 & 1.000 & 1.000 & 1.000 \\
\hline$\hat{\hat{y}}_{t}$ & 1.000 & 1.000 & 1.000 & 0.994 & 1.000 & 1.000 & 1.000 & 1.000 & 1.000 & 1.000 & 1.000 & 1.000 \\
\hline$\hat{\hat{u}}_{t}$ & 1.000 & 1.000 & 1.000 & 0.996 & 1.000 & 1.000 & 0.996 & 0.998 & 1.000 & 1.000 & 1.000 & 1.000 \\
\hline$\hat{i_{t}}$ & 1.000 & 1.000 & 0.980 & 0.982 & 0.993 & 0.993 & 1.000 & 1.000 & 1.000 & 1.000 & 1.000 & 1.000 \\
\hline
\end{tabular}

Note: Reports correlations with the central estimates.

\section{CONClusion}

This paper considers the problem of jointly decomposing a set of time series variables into cyclical and trend components, subject to sets of stochastic linear restrictions among these cyclical and trend components. We derive a closed form solution to an ordinary problem featuring homogeneous penalty term difference orders and static restrictions, as well as to a generalized problem featuring heterogeneous penalty term difference orders and dynamic restrictions. We use our GMLF to jointly estimate potential output, the natural rate of unemployment and the natural rate of interest, conditional on selected equilibrium conditions from a calibrated New Keynesian model.

Several properties of the unobserved components estimates from the closed form multivariate linear filters proposed in this paper remain to be established. Do they correspond to those from possibly restricted linear unobserved components models? What distributions are they drawn from under alternative assumptions? How do they compare to those from possibly misspecified linear unobserved components models in terms of empirical performance and computational cost? How much do they get revised as the sample expands? These questions remain to be answered by future research.

\section{Appendix A. Proof of Proposition 1}

The Ordinary Multivariate Linear Filter (OMLF) solves the following minimization problem, where $y_{i, t}=\hat{y}_{i, t}+\bar{y}_{i, t}$, while $G \leq N$ and $H \leq N$ :

$$
\min _{\left\{\left\{\bar{y}_{i, t}\right\}_{i=1}^{N}\right\}_{t=1}^{T}} S\left(\left\{\left\{\bar{y}_{i, t}\right\}_{i=1}^{N}\right\}_{t=1}^{T}\right)=\sum_{i=1}^{N} \sum_{t=1}^{T} \hat{y}_{i, t}^{2}+\lambda^{2} \sum_{i=1}^{N} \sum_{t=d+1}^{T}\left(\Delta^{d} \bar{y}_{i, t}\right)^{2}+\gamma^{2} \sum_{g=1}^{G} \sum_{t=1}^{T}\left(\sum_{i=1}^{N} \phi_{g, i} \hat{y}_{i, t}\right)^{2}+\psi^{2} \sum_{h=1}^{H} \sum_{t=1}^{T}\left(\sum_{i=1}^{N} \theta_{h, i} \bar{y}_{i, t}\right)^{2}
$$


Using matrix notation, this minimization problem may be restated as follows, where $\boldsymbol{y}_{i}=\hat{\boldsymbol{y}}_{i}+\overline{\boldsymbol{y}}_{i}$ and $\boldsymbol{Y}=\hat{\boldsymbol{Y}}+\overline{\boldsymbol{Y}}$, while $\boldsymbol{\Delta}^{d}=\prod_{i=1}^{d}\left(\left[\begin{array}{ll}\mathbf{0} & \boldsymbol{I}_{T-i}\end{array}\right]-\left[\begin{array}{ll}\boldsymbol{I}_{T-i} & \mathbf{0}\end{array}\right]\right)$ :

$$
\begin{aligned}
& \sum_{i=1}^{N} \sum_{t=1}^{T} \hat{y}_{i, t}^{2}=\sum_{i=1}^{N} \hat{\boldsymbol{y}}_{i}^{\top} \hat{\boldsymbol{y}}_{i}=\operatorname{Tr}\left(\left[\begin{array}{ccc}
\hat{\boldsymbol{y}}_{1}^{\top} \hat{\boldsymbol{y}}_{1} & \cdots & \hat{\boldsymbol{y}}_{1}^{\top} \hat{\boldsymbol{y}}_{N} \\
\vdots & \ddots & \vdots \\
\hat{\boldsymbol{y}}_{N}^{\top} \hat{\boldsymbol{y}}_{1} & \cdots & \hat{\boldsymbol{y}}_{N}^{\top} \hat{\boldsymbol{y}}_{N}
\end{array}\right]\right)=\operatorname{Tr}\left(\left[\begin{array}{c}
\hat{\boldsymbol{y}}_{1}^{\top} \\
\vdots \\
\hat{\boldsymbol{y}}_{N}^{\top}
\end{array}\right]\left[\begin{array}{lll}
\hat{\boldsymbol{y}}_{1} & \cdots & \hat{\boldsymbol{y}}_{N}
\end{array}\right]\right) \operatorname{Tr}\left(\hat{\boldsymbol{Y}}^{\top} \hat{\boldsymbol{Y}}\right) \\
& \lambda^{2} \sum_{i=1}^{N} \sum_{t=d+1}^{T}\left(\Delta^{d} \bar{y}_{i, t}\right)^{2}=\lambda^{2} \sum_{i=1}^{N}\left(\boldsymbol{\Delta}^{d} \overline{\boldsymbol{y}}_{i}\right)^{\top}\left(\boldsymbol{\Delta}^{d} \overline{\boldsymbol{y}}_{i}\right)=\lambda^{2} \operatorname{Tr}\left(\left[\begin{array}{ccc}
\left(\boldsymbol{\Delta}^{d} \overline{\boldsymbol{y}}_{1}\right)^{\top}\left(\boldsymbol{\Delta}^{d} \overline{\boldsymbol{y}}_{1}\right) & \cdots & \left(\boldsymbol{\Delta}^{d} \overline{\boldsymbol{y}}_{1}\right)^{\top}\left(\boldsymbol{\Delta}^{d} \overline{\boldsymbol{y}}_{N}\right) \\
\vdots & \ddots & \vdots \\
\left(\boldsymbol{\Delta}^{d} \overline{\boldsymbol{y}}_{N}\right)^{\top}\left(\boldsymbol{\Delta}^{d} \overline{\boldsymbol{y}}_{1}\right) & \cdots & \left(\boldsymbol{\Delta}^{d} \overline{\boldsymbol{y}}_{N}\right)^{\top}\left(\boldsymbol{\Delta}^{d} \overline{\boldsymbol{y}}_{N}\right)
\end{array}\right]\right)
\end{aligned}
$$

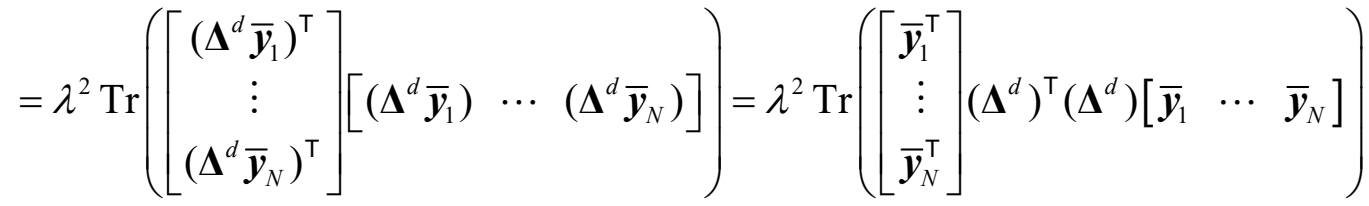

$$
\begin{aligned}
& =\lambda^{2} \operatorname{Tr}\left(\overline{\boldsymbol{Y}}^{\top}\left(\boldsymbol{\Delta}^{d}\right)^{\top}\left(\boldsymbol{\Delta}^{d}\right) \overline{\boldsymbol{Y}}\right)=\lambda^{2} \operatorname{Tr}\left(\left(\boldsymbol{\Delta}^{d} \overline{\boldsymbol{Y}}\right)^{\top}\left(\boldsymbol{\Delta}^{d} \overline{\boldsymbol{Y}}\right)\right) \\
& \gamma^{2} \sum_{g=1}^{G} \sum_{t=1}^{T}\left(\sum_{i=1}^{N} \phi_{g, i} \hat{y}_{i, t}\right)^{2}=\gamma^{2} \sum_{g=1}^{G}\left(\sum_{i=1}^{N} \phi_{g, i} \hat{\boldsymbol{y}}_{i}\right)^{\top}\left(\sum_{i=1}^{N} \phi_{g, i} \hat{\boldsymbol{y}}_{i}\right)=\gamma^{2} \sum_{g=1}^{G}\left(\hat{\boldsymbol{Y}} \boldsymbol{\phi}_{g}\right)^{\top}\left(\hat{\boldsymbol{Y}} \boldsymbol{\phi}_{g}\right) \\
& =\gamma^{2} \operatorname{Tr}\left(\left[\begin{array}{ccc}
(\hat{\boldsymbol{Y}} \boldsymbol{\phi})^{\top}(\hat{\boldsymbol{Y}} \boldsymbol{\phi}) & \cdots & (\hat{\boldsymbol{Y}} \boldsymbol{\phi})^{\top}\left(\hat{\boldsymbol{Y}} \boldsymbol{\phi}_{G}\right) \\
\vdots & \ddots & \vdots \\
\left(\hat{\boldsymbol{Y}} \boldsymbol{\phi}_{G}\right)^{\top}(\hat{\boldsymbol{Y}} \boldsymbol{\phi}) & \cdots & \left(\hat{\boldsymbol{Y}} \boldsymbol{\phi}_{G}\right)^{\top}\left(\hat{\boldsymbol{Y}} \boldsymbol{\phi}_{G}\right)
\end{array}\right]\right)=\gamma^{2} \operatorname{Tr}\left(\left[\begin{array}{c}
(\hat{\boldsymbol{Y}} \boldsymbol{\phi})^{\top} \\
\vdots \\
\left(\hat{\boldsymbol{Y}} \boldsymbol{\phi}_{G}\right)^{\top}
\end{array}\right]\left[\begin{array}{lll}
(\hat{\boldsymbol{Y}} \boldsymbol{\phi}) & \cdots & \left(\hat{\boldsymbol{Y}} \boldsymbol{\phi}_{G}\right)
\end{array}\right]\right) \\
& =\gamma^{2} \operatorname{Tr}\left(\left[\begin{array}{c}
\boldsymbol{\phi}^{\top} \\
\vdots \\
\boldsymbol{\phi}_{G}^{\top}
\end{array}\right] \hat{\boldsymbol{Y}}^{\top} \hat{\boldsymbol{Y}}\left[\begin{array}{lll}
\boldsymbol{\phi} & \cdots & \boldsymbol{\phi}_{G}
\end{array}\right]\right)=\gamma^{2} \operatorname{Tr}\left(\boldsymbol{\Phi}^{\top} \hat{\boldsymbol{Y}}^{\top} \hat{\boldsymbol{Y}} \boldsymbol{\Phi}\right)=\gamma^{2} \operatorname{Tr}\left((\hat{\boldsymbol{Y}} \boldsymbol{\Phi})^{\top}(\hat{\boldsymbol{Y}} \boldsymbol{\Phi})\right) \\
& \psi^{2} \sum_{h=1}^{H} \sum_{t=1}^{T}\left(\sum_{i=1}^{N} \theta_{h, i} \bar{y}_{i, t}\right)^{2}=\psi^{2} \sum_{h=1}^{H}\left(\sum_{i=1}^{N} \theta_{h, i} \overline{\boldsymbol{y}}_{i}\right)^{\top}\left(\sum_{i=1}^{N} \theta_{h, i} \overline{\boldsymbol{y}}_{i}\right)=\psi^{2} \sum_{h=1}^{H}\left(\overline{\boldsymbol{Y}} \boldsymbol{\theta}_{h}\right)^{\top}\left(\overline{\boldsymbol{Y}} \boldsymbol{\theta}_{h}\right)
\end{aligned}
$$

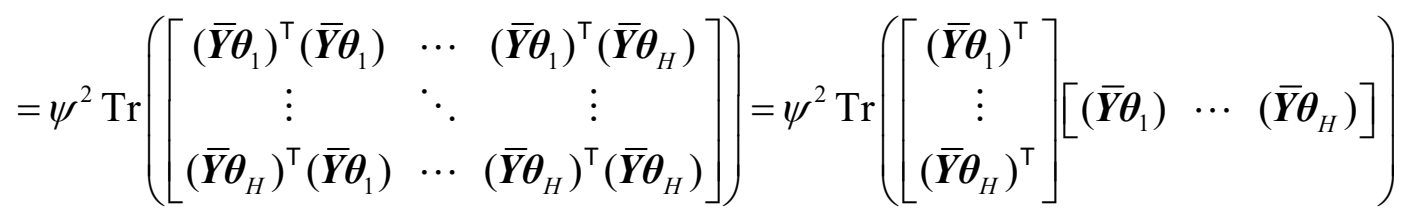

$$
\begin{aligned}
& =\psi^{2} \operatorname{Tr}\left(\left[\begin{array}{c}
\boldsymbol{\theta}_{1}^{\top} \\
\vdots \\
\boldsymbol{\theta}_{H}^{\top}
\end{array}\right] \overline{\boldsymbol{Y}}^{\top} \overline{\boldsymbol{Y}}\left[\begin{array}{lll}
\boldsymbol{\theta}_{1} & \cdots & \boldsymbol{\theta}_{H}
\end{array}\right]\right)=\psi^{2} \operatorname{Tr}\left(\boldsymbol{\Theta}^{\top} \overline{\boldsymbol{Y}}^{\top} \overline{\boldsymbol{Y}} \boldsymbol{\Theta}\right)=\psi^{2} \operatorname{Tr}\left((\overline{\boldsymbol{Y}} \boldsymbol{\Theta})^{\top}(\overline{\boldsymbol{Y}} \boldsymbol{\Theta})\right)
\end{aligned}
$$$$
\min _{\overline{\boldsymbol{Y}}} S(\overline{\boldsymbol{Y}})=\operatorname{Tr}\left(\hat{\boldsymbol{Y}}^{\top} \hat{\boldsymbol{Y}}\right)+\lambda^{2} \operatorname{Tr}\left(\left(\boldsymbol{\Delta}^{d} \overline{\boldsymbol{Y}}\right)^{\top}\left(\boldsymbol{\Delta}^{d} \overline{\boldsymbol{Y}}\right)\right)+\gamma^{2} \operatorname{Tr}\left((\hat{\boldsymbol{Y}} \boldsymbol{\Phi})^{\top}(\hat{\boldsymbol{Y}} \boldsymbol{\Phi})\right)+\psi^{2} \operatorname{Tr}\left((\overline{\boldsymbol{Y}} \boldsymbol{\Theta})^{\top}(\overline{\boldsymbol{Y}} \boldsymbol{\Theta})\right)
$$ 
The necessary first order condition for a local extremum at $\overline{\boldsymbol{Y}}_{\mid T}$ yields:

$$
\begin{aligned}
& \frac{\partial S(\overline{\boldsymbol{Y}})}{\partial \overline{\boldsymbol{Y}}}=-2\left[\hat{\boldsymbol{Y}}-\lambda^{2}\left(\boldsymbol{\Delta}^{d}\right)^{\top}\left(\boldsymbol{\Delta}^{d}\right) \overline{\boldsymbol{Y}}+\gamma^{2} \hat{\boldsymbol{Y}} \boldsymbol{\Phi} \boldsymbol{\Phi}^{\top}-\psi^{2} \overline{\boldsymbol{Y}} \boldsymbol{\Theta} \boldsymbol{\Theta}^{\top}\right]=\mathbf{0} \\
& \Rightarrow \overline{\boldsymbol{Y}}+\lambda^{2}\left(\boldsymbol{\Delta}^{d}\right)^{\top}\left(\boldsymbol{\Delta}^{d}\right) \overline{\boldsymbol{Y}}+\gamma^{2} \overline{\boldsymbol{Y}} \boldsymbol{\Phi} \boldsymbol{\Phi}^{\top}+\psi^{2} \overline{\boldsymbol{Y}} \boldsymbol{\Theta} \boldsymbol{\Theta}^{\top}=\boldsymbol{Y}+\gamma^{2} \boldsymbol{Y} \boldsymbol{\Phi} \boldsymbol{\Phi}^{\top} \\
& \Rightarrow \operatorname{Vec}\left(\overline{\boldsymbol{Y}}_{\mid T}\right)=\left[\boldsymbol{I}_{N T}+\left(\boldsymbol{I}_{N} \otimes\left(\lambda^{2}\left(\boldsymbol{\Delta}^{d}\right)^{\top}\left(\boldsymbol{\Delta}^{d}\right)\right)\right)+\left(\left(\gamma^{2} \boldsymbol{\Phi} \boldsymbol{\Phi}^{\top}+\psi^{2} \boldsymbol{\Theta} \boldsymbol{\Theta}^{\boldsymbol{\top}}\right) \otimes \boldsymbol{I}_{T}\right)\right]^{-1}\left[\boldsymbol{I}_{N T}+\left(\left(\gamma^{2} \boldsymbol{\Phi} \boldsymbol{\Phi}^{\boldsymbol{\top}}\right) \otimes \boldsymbol{I}_{T}\right)\right] \operatorname{Vec}(\boldsymbol{Y})
\end{aligned}
$$

The sufficient second order condition for a unique global minimum at $\overline{\boldsymbol{Y}}_{\mid T}$ is satisfied:

$$
\begin{aligned}
& \frac{\partial^{2} S(\overline{\boldsymbol{Y}})}{\partial \overline{\boldsymbol{Y}} \partial \overline{\boldsymbol{Y}}^{\boldsymbol{\top}}}=-2 \frac{\partial}{\partial \operatorname{Vec}(\overline{\boldsymbol{Y}})^{\top}}\left[\operatorname{Vec}(\hat{\boldsymbol{Y}})-\left(\boldsymbol{I}_{N} \otimes\left(\lambda^{2}\left(\boldsymbol{\Delta}^{d}\right)^{\top}\left(\boldsymbol{\Delta}^{d}\right)\right)\right) \operatorname{Vec}(\overline{\boldsymbol{Y}})+\left(\left(\gamma^{2} \boldsymbol{\Phi} \boldsymbol{\Phi}^{\boldsymbol{\top}}\right) \otimes \boldsymbol{I}_{T}\right) \operatorname{Vec}(\hat{\boldsymbol{Y}})-\left(\left(\psi^{2} \boldsymbol{\Theta} \boldsymbol{\Theta}^{\boldsymbol{\top}}\right) \otimes \boldsymbol{I}_{T}\right) \operatorname{Vec}(\overline{\boldsymbol{Y}})\right] \\
& =2\left[\boldsymbol{I}_{N T}+\left(\boldsymbol{I}_{N} \otimes\left(\lambda^{2}\left(\boldsymbol{\Delta}^{d}\right)^{\top}\left(\boldsymbol{\Delta}^{d}\right)\right)\right)+\left(\left(\gamma^{2} \boldsymbol{\Phi} \boldsymbol{\Phi}^{\top}+\psi^{2} \boldsymbol{\Theta} \boldsymbol{\Theta}^{\boldsymbol{\top}}\right) \otimes \boldsymbol{I}_{T}\right)\right]
\end{aligned}
$$

The Hessian matrix of the objective function $\frac{\partial^{2} S(\overline{\boldsymbol{Y}})}{\partial \overline{\boldsymbol{Y}} \partial \overline{\boldsymbol{Y}}^{\top}}$ is positive definite throughout its domain, because: i) the sum of positive definite and positive semidefinite matrices is positive definite; ii) the identity matrix is positive definite; iii) the Kronecker product of positive semidefinite matrices is positive semidefinite; and iv) the product of a matrix and its transpose is positive semidefinite. It follows that $\overline{\boldsymbol{Y}}_{\mid T}$ exists, because a positive definite matrix is nonsingular.

\section{Appendix B. Proof of Proposition 2}

The Generalized Multivariate Linear Filter (GMLF) solves the following minimization problem, where $y_{i, t}=\hat{y}_{i, t}+\bar{y}_{i, t}$, while $\lambda_{d, i}=0$ for all $d \neq d_{i}$, with $G \leq N$ and $H \leq N$ :

$$
\min _{\left\{\left\{\bar{y}_{i, t}, i_{i=1}^{T}\right\}_{t=1}\right.} S\left(\left\{\left\{\bar{y}_{i, t}\right\}_{i=1}^{N}\right\}_{t=1}^{T}\right)=\sum_{i=1}^{N} \sum_{t=1}^{T} \hat{y}_{i, t}^{2}+\sum_{i=1}^{N} \sum_{d=1}^{D} \lambda_{d, i}^{2} \sum_{t=d+1}^{T}\left(\Delta^{d} \bar{y}_{i, t}\right)^{2}+\sum_{g=1}^{G} \gamma_{g}^{2} \sum_{t=P_{1}+1}^{T-P_{2}}\left(\sum_{i=1}^{N} \sum_{p=-P_{1}}^{P_{2}} \phi_{g, i, p} \hat{y}_{i, t+p}\right)^{2}+\sum_{h=1}^{H} \psi_{h}^{2} \sum_{t=Q_{1}+1}^{T-Q_{2}}\left(\sum_{i=1}^{N} \sum_{q=-Q_{1}}^{Q_{2}} \theta_{h, i, q} \bar{y}_{i, t+q}\right)^{2}
$$

Using matrix notation, this minimization problem may be restated as follows, where $\boldsymbol{y}_{i}=\hat{\boldsymbol{y}}_{i}+\overline{\boldsymbol{y}}_{\dot{j}}$ and $\quad \boldsymbol{Y}=\hat{\boldsymbol{Y}}+\overline{\boldsymbol{Y}}, \quad$ while $\quad \boldsymbol{\Delta}^{d}=\prod_{i=1}^{d}\left(\left[\begin{array}{ll}\mathbf{0} & \boldsymbol{I}_{T-i}\end{array}\right]-\left[\begin{array}{ll}\boldsymbol{I}_{T=i} & \mathbf{0}\end{array}\right]\right), \quad$ with $\quad \boldsymbol{L}_{\boldsymbol{P}}^{p}=\left[\begin{array}{ll}\boldsymbol{I}_{T-P_{1}-P_{2}} & \mathbf{0}\end{array}\right]$ $\left[\begin{array}{ll}\mathbf{0} & \boldsymbol{I}_{T-P_{1}}\end{array}\right]\left[\begin{array}{ll}\boldsymbol{I}_{T-p} & \mathbf{0}\end{array}\right]$ if $p \leq 0$ and $\boldsymbol{L}_{\boldsymbol{P}}^{p}=\left[\begin{array}{ll}\mathbf{0} & \boldsymbol{I}_{T-P_{1}-P_{2}}\end{array}\right]\left[\begin{array}{ll}\boldsymbol{I}_{T-P_{2}} & \mathbf{0}\end{array}\right]\left[\begin{array}{ll}\mathbf{0} & \boldsymbol{I}_{T-p}\end{array}\right]$ otherwise, and $\boldsymbol{L}_{\boldsymbol{Q}}^{q}=\left[\begin{array}{cc}\boldsymbol{I}_{T-Q_{1}-Q_{2}} & \mathbf{0}\end{array}\right]\left[\begin{array}{ll}\mathbf{0} & \boldsymbol{I}_{T-Q_{1}}\end{array}\right]\left[\begin{array}{ll}\boldsymbol{I}_{T-q} & \mathbf{0}\end{array}\right]$ if $q \leq 0$ and $\boldsymbol{L}_{\boldsymbol{Q}}^{q}=\left[\begin{array}{ll}\mathbf{0} & \boldsymbol{I}_{T-Q_{1}-Q_{2}}\end{array}\right]\left[\begin{array}{ll}\boldsymbol{I}_{T-Q_{2}} & \mathbf{0}\end{array}\right]\left[\begin{array}{ll}\mathbf{0} & \boldsymbol{I}_{T-q}\end{array}\right]$ otherwise:

$$
\sum_{i=1}^{N} \sum_{t=1}^{T} \hat{y}_{i, t}^{2}=\sum_{i=1}^{N} \hat{\boldsymbol{y}}_{i}^{\top} \hat{\boldsymbol{y}}_{i}=\operatorname{Tr}\left(\left[\begin{array}{ccc}
\hat{\boldsymbol{y}}_{1}^{\top} \hat{\boldsymbol{y}}_{1} & \cdots & \hat{\boldsymbol{y}}_{1}^{\top} \hat{\boldsymbol{y}}_{N} \\
\vdots & \ddots & \vdots \\
\hat{\boldsymbol{y}}_{N}^{\top} \hat{\boldsymbol{y}}_{1} & \cdots & \hat{\boldsymbol{y}}_{N}^{\top} \hat{\boldsymbol{y}}_{N}
\end{array}\right]\right)=\operatorname{Tr}\left(\left[\begin{array}{c}
\hat{\boldsymbol{y}}_{1}^{\top} \\
\vdots \\
\hat{\boldsymbol{y}}_{N}^{\top}
\end{array}\right]\left[\begin{array}{lll}
\hat{\boldsymbol{y}}_{1} & \cdots & \hat{\boldsymbol{y}}_{N}
\end{array}\right]\right) \operatorname{Tr}\left(\hat{\boldsymbol{Y}}^{\top} \hat{\boldsymbol{Y}}\right)
$$




$$
\begin{aligned}
& \sum_{i=1}^{N} \sum_{d=1}^{D} \lambda_{d, i}^{2} \sum_{t=d+1}^{T}\left(\Delta^{d} \bar{y}_{i, t}\right)^{2}=\sum_{d=1}^{D} \sum_{i=1}^{N} \lambda_{d, i}^{\top}\left(\Delta^{d} \overline{\boldsymbol{y}}_{i}\right)^{\top}\left(\boldsymbol{\Delta}^{d} \overline{\boldsymbol{y}}_{i}\right) \lambda_{d, i}=\sum_{d=1}^{D} \operatorname{Tr}\left(\left[\begin{array}{ccc}
\lambda_{d, 1}^{\top}\left(\boldsymbol{\Delta}^{d} \overline{\boldsymbol{y}}_{1}\right)^{\top}\left(\boldsymbol{\Delta}^{d} \overline{\boldsymbol{y}}_{1}\right) \lambda_{d, 1} & \cdots & \lambda_{d, 1}^{\top}\left(\boldsymbol{\Delta}^{d} \overline{\boldsymbol{y}}_{1}\right)^{\top}\left(\boldsymbol{\Delta}^{d} \overline{\boldsymbol{y}}_{N}\right) \lambda_{d, N} \\
\vdots & \ddots & \vdots \\
\lambda_{d, N}^{\top}\left(\boldsymbol{\Delta}^{d} \overline{\boldsymbol{y}}_{N}\right)^{\top}\left(\boldsymbol{\Delta}^{d} \overline{\boldsymbol{y}}_{1}\right) \lambda_{d, 1} & \cdots & \lambda_{d, N}^{\top}\left(\boldsymbol{\Delta}^{d} \overline{\boldsymbol{y}}_{N}\right)^{\top}\left(\boldsymbol{\Delta}^{d} \overline{\boldsymbol{y}}_{N}\right) \lambda_{d, N}
\end{array}\right]\right) \\
& =\sum_{d=1}^{D} \operatorname{Tr}\left(\left[\begin{array}{ccc}
\lambda_{d, 1}^{\top} & \cdots & 0 \\
\vdots & \ddots & \vdots \\
0 & \cdots & \lambda_{d, N}^{\top}
\end{array}\right]\left[\begin{array}{ccc}
\left(\boldsymbol{\Delta}^{d} \overline{\boldsymbol{y}}_{1}\right)^{\top}\left(\boldsymbol{\Delta}^{d} \overline{\boldsymbol{y}}_{1}\right) & \cdots & \left(\boldsymbol{\Delta}^{d} \overline{\boldsymbol{y}}_{1}\right)^{\top}\left(\boldsymbol{\Delta}^{d} \overline{\boldsymbol{y}}_{N}\right) \\
\vdots & \ddots & \vdots \\
\left(\boldsymbol{\Delta}^{d} \overline{\boldsymbol{y}}_{N}\right)^{\top}\left(\boldsymbol{\Delta}^{d} \overline{\boldsymbol{y}}_{1}\right) & \cdots & \left(\boldsymbol{\Delta}^{d} \overline{\boldsymbol{y}}_{N}\right)^{\top}\left(\boldsymbol{\Delta}^{d} \overline{\boldsymbol{y}}_{N}\right)
\end{array}\right]\left[\begin{array}{ccc}
\lambda_{d, 1} & \cdots & 0 \\
\vdots & \ddots & \vdots \\
0 & \cdots & \lambda_{d, N}
\end{array}\right]\right)
\end{aligned}
$$

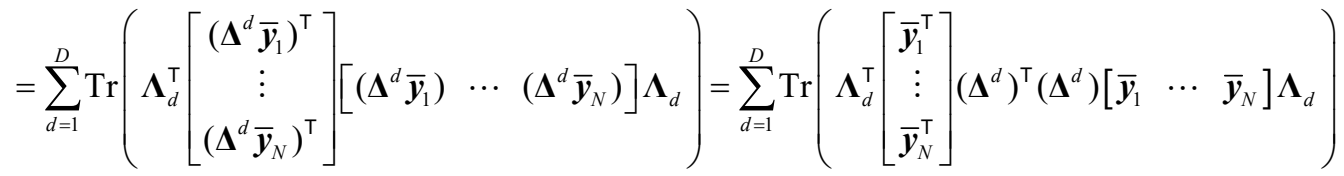

$$
\begin{aligned}
& =\sum_{d=1}^{D} \operatorname{Tr}\left(\boldsymbol{\Lambda}_{d}^{\top} \overline{\boldsymbol{Y}}^{\top}\left(\boldsymbol{\Delta}^{d}\right)^{\top}\left(\boldsymbol{\Delta}^{d}\right) \overline{\boldsymbol{Y}} \boldsymbol{\Lambda}_{d}\right)=\sum_{d=1}^{D} \operatorname{Tr}\left(\left(\boldsymbol{\Delta}^{d} \overline{\boldsymbol{Y}} \boldsymbol{\Lambda}_{d}\right)^{\top}\left(\boldsymbol{\Delta}^{d} \overline{\boldsymbol{Y}} \boldsymbol{\Lambda}_{d}\right)\right) \\
& \sum_{g=1}^{G} \gamma_{g}^{2} \sum_{t=P_{1}+1}^{T-P_{2}}\left(\sum_{i=1}^{N} \sum_{p=-P_{1}}^{P_{2}} \phi_{g, i, p} \hat{y}_{i, t+p}\right)^{2}=\sum_{g=1}^{G} \gamma_{g}^{2} \sum_{t=P_{1}+1}^{T-P_{2}}\left(\sum_{p=-P_{1}}^{P_{2}} L^{p} \sum_{i=1}^{N} \phi_{g, i, p} \hat{y}_{i, t}\right)^{2}=\sum_{g=1}^{G} \gamma_{g}^{\top}\left(\sum_{p=-P_{1}}^{P_{2}} \boldsymbol{L}_{P}^{p} \sum_{i=1}^{N} \phi_{g, i, p} \hat{\boldsymbol{y}}_{i}\right)^{\top}\left(\sum_{p=-P_{1}}^{P_{2}} \boldsymbol{L}_{P}^{p} \sum_{i=1}^{N} \boldsymbol{\phi}_{g, i, p} \hat{\boldsymbol{y}}_{i}\right) \gamma_{g}
\end{aligned}
$$

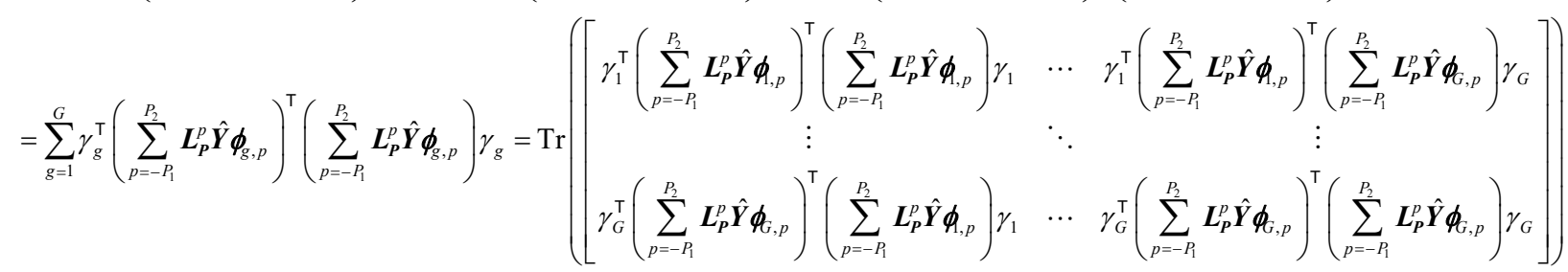

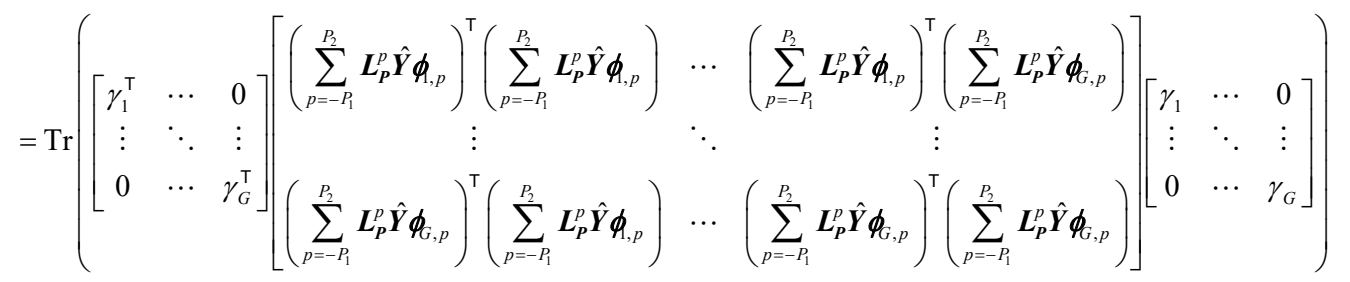

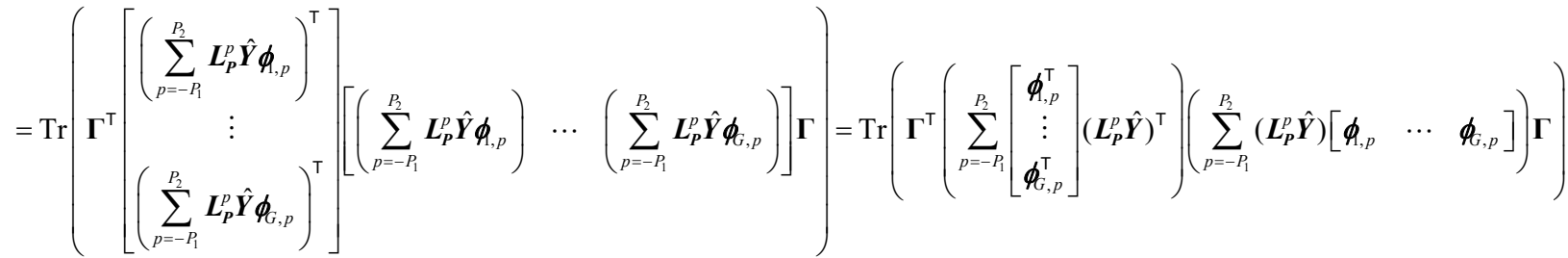

$$
\begin{aligned}
& =\operatorname{Tr}\left(\boldsymbol{\Gamma}^{\top}\left(\sum_{p=-P_{1}}^{P_{2}} \boldsymbol{L}_{P}^{p} \hat{\boldsymbol{Y}} \boldsymbol{\Phi}_{p}\right)^{\top}\left(\sum_{p=-P_{1}}^{P_{2}} \boldsymbol{L}_{P}^{p} \hat{\boldsymbol{Y}} \boldsymbol{\Phi}_{p}\right) \boldsymbol{\Gamma}\right)=\operatorname{Tr}\left(\left(\sum_{p=-P_{1}}^{P_{2}} \boldsymbol{L}_{P}^{p} \hat{\boldsymbol{Y}} \boldsymbol{\Phi}_{p} \boldsymbol{\Gamma}\right)^{\top}\left(\sum_{p=-P_{1}}^{P_{2}} \boldsymbol{L}_{P}^{p} \hat{\boldsymbol{Y}} \boldsymbol{\Phi}_{p} \boldsymbol{\Gamma}\right)\right)
\end{aligned}
$$




$$
\begin{aligned}
& \sum_{h=1}^{H} \psi_{h}^{2} \sum_{t=Q_{1}+1}^{T-Q_{2}}\left(\sum_{i=1}^{N} \sum_{q=-Q_{1}}^{Q_{2}} \theta_{h, i, q} \bar{y}_{i, t+q}\right)^{2}=\sum_{h=1}^{H} \psi_{h}^{2} \sum_{t=Q_{1}+1}^{T-Q_{2}}\left(\sum_{q=-Q_{1}}^{Q_{2}} L^{q} \sum_{i=1}^{N} \theta_{h, i, q} \bar{y}_{i, t}\right)^{2}=\sum_{h=1}^{H} \psi_{h}^{\top}\left(\sum_{q=-Q_{1}}^{Q_{2}} \boldsymbol{L}_{Q}^{q} \sum_{i=1}^{N} \theta_{h, i, q} \overline{\boldsymbol{y}}_{i}\right)^{\top}\left(\sum_{q=-Q_{1}}^{Q_{2}} \boldsymbol{L}_{Q}^{q} \sum_{i=1}^{N} \theta_{h, i, q} \overline{\boldsymbol{y}}_{i}\right) \psi_{h}
\end{aligned}
$$

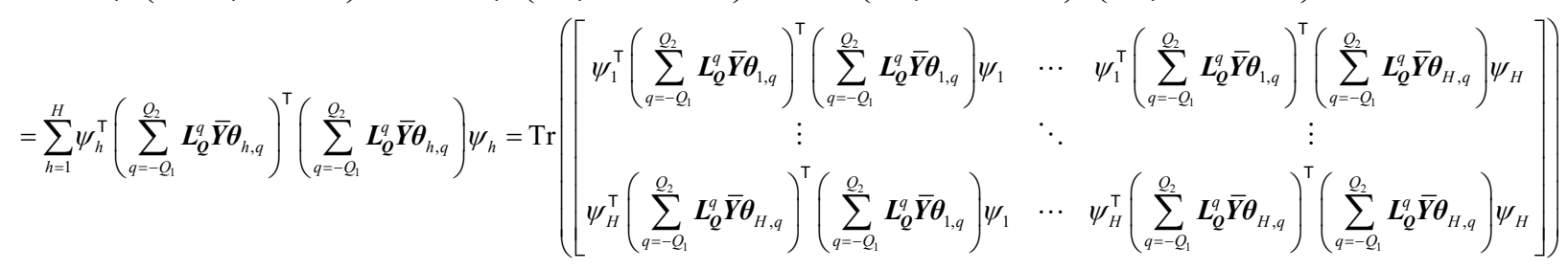

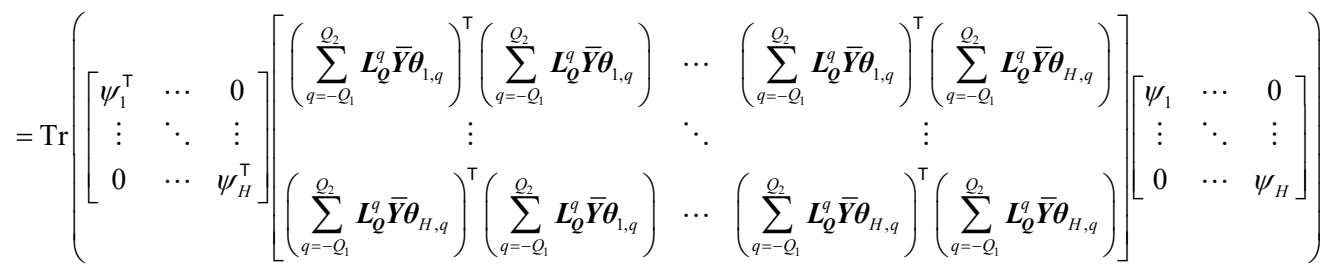

$$
\begin{aligned}
& \left.=\operatorname{Tr}\left(\boldsymbol{\Psi}^{\top}\left[\begin{array}{c}
\left(\sum_{q=-Q_{1}}^{Q_{2}} \boldsymbol{L}_{\boldsymbol{Q}}^{q} \overline{\boldsymbol{Y}} \boldsymbol{\theta}_{1, q}\right. \\
\vdots \\
\left(\sum_{q=-Q_{1}}^{Q_{2}} \boldsymbol{L}_{\boldsymbol{Q}}^{q} \overline{\boldsymbol{Y}} \boldsymbol{\theta}_{H, q}\right.
\end{array}\right)^{\top}\right]\left[\left(\sum_{q=-Q_{1}}^{Q_{2}} \boldsymbol{L}_{Q}^{q} \overline{\boldsymbol{Y}} \boldsymbol{\theta}_{1, q}\right) \cdots\left(\sum_{q=-Q_{1}}^{Q_{2}} \boldsymbol{L}_{Q}^{q} \overline{\boldsymbol{Y}} \boldsymbol{\theta}_{H, q}\right)\right] \boldsymbol{\Psi}\right)=\operatorname{Tr}\left(\boldsymbol{\Psi}^{\top}\left(\sum_{q=Q_{1}}^{Q_{2}}\left[\begin{array}{c}
\boldsymbol{\theta}_{1, q}^{\top} \\
\vdots \\
\boldsymbol{\theta}_{H, q}^{\top}
\end{array}\right]\left(\boldsymbol{L}_{Q}^{q} \overline{\boldsymbol{Y}}\right)^{\top}\right)\left(\sum_{q=-Q_{1}}^{Q_{2}}\left(\boldsymbol{L}_{Q}^{q} \overline{\boldsymbol{Y}}\right)\left[\begin{array}{lll}
\boldsymbol{\theta}_{1, q} & \cdots & \boldsymbol{\theta}_{H, q}
\end{array}\right]\right) \boldsymbol{\Psi}\right) \\
& =\operatorname{Tr}\left(\boldsymbol{\Psi}^{\top}\left(\sum_{q=-Q_{1}}^{Q_{2}} \boldsymbol{L}_{Q}^{q} \overline{\mathbf{Y}} \mathbf{\Theta}_{q}\right)^{\top}\left(\sum_{q=-Q_{1}}^{Q_{2}} \boldsymbol{L}_{Q}^{q} \overline{\mathbf{Y}} \mathbf{\Theta}_{q}\right) \boldsymbol{\Psi}\right)=\operatorname{Tr}\left(\left(\sum_{q=-Q_{1}}^{Q_{2}} \boldsymbol{L}_{Q}^{q} \overline{\mathbf{Y}} \mathbf{\Theta}_{q} \boldsymbol{\Psi}\right)^{\top}\left(\sum_{q=-Q_{1}}^{Q_{2}} \boldsymbol{L}_{Q}^{q} \overline{\mathbf{Y}} \mathbf{\Theta}_{q} \boldsymbol{\Psi}\right)\right) \\
& \min _{\bar{Y}} S(\overline{\boldsymbol{Y}})=\operatorname{Tr}\left(\hat{\boldsymbol{Y}}^{\top} \hat{\boldsymbol{Y}}\right)+\sum_{d=1}^{D} \operatorname{Tr}\left(\left(\boldsymbol{\Delta}^{d} \overline{\boldsymbol{Y}} \boldsymbol{\Lambda}_{d}\right)^{\top}\left(\boldsymbol{\Delta}^{d} \overline{\boldsymbol{Y}} \boldsymbol{\Lambda}_{d}\right)\right)+\operatorname{Tr}\left(\left(\sum_{p=-P_{1}}^{P_{2}} \boldsymbol{L}_{\boldsymbol{P}}^{p} \hat{\boldsymbol{Y}} \boldsymbol{\Phi}_{p} \boldsymbol{\Gamma}\right)^{\top}\left(\sum_{p=-P_{1}}^{P_{2}} \boldsymbol{L}_{\boldsymbol{P}}^{p} \hat{\boldsymbol{Y}} \boldsymbol{\Phi}_{p} \boldsymbol{\Gamma}\right)\right)+\operatorname{Tr}\left(\left(\sum_{q=-Q_{1}}^{Q_{2}} \boldsymbol{L}_{Q}^{q} \overline{\boldsymbol{Y}} \boldsymbol{\Theta}_{q} \boldsymbol{\Psi}\right)^{\top}\left(\sum_{q=-Q_{1}}^{Q_{2}} \boldsymbol{L}_{\boldsymbol{Q}}^{q} \overline{\boldsymbol{Y}} \boldsymbol{\Theta}_{q} \boldsymbol{\Psi}\right)\right)
\end{aligned}
$$

The necessary first order condition for a local extremum at $\overline{\boldsymbol{Y}}_{\mid T}$ yields:

$\frac{\partial S(\overline{\boldsymbol{Y}})}{\partial \overline{\boldsymbol{Y}}}=-2\left[\hat{\boldsymbol{Y}}-\sum_{d=1}^{D}\left(\boldsymbol{\Delta}^{d}\right)^{\top}\left(\boldsymbol{\Delta}^{d}\right) \overline{\boldsymbol{Y}} \boldsymbol{\Lambda}_{d} \boldsymbol{\Lambda}_{d}^{\top}+\sum_{p=-p_{p}}^{p_{3}}\left(\boldsymbol{L}_{p}^{p}\right)^{\top}\left(\boldsymbol{L}_{p}^{p}\right) \hat{\boldsymbol{Y}}\left(\boldsymbol{\Phi}_{p} \boldsymbol{\Gamma}\right)\left(\boldsymbol{\Phi}_{p} \boldsymbol{\Gamma}\right)^{\top}-\sum_{q=-Q_{1}}^{Q_{2}}\left(\boldsymbol{L}_{Q}^{q}\right)^{\top}\left(\boldsymbol{L}_{Q}^{q}\right) \overline{\boldsymbol{Y}}\left(\boldsymbol{\Theta}_{q} \boldsymbol{\Psi}\right)\left(\boldsymbol{\Theta}_{q} \boldsymbol{\Psi}\right)^{\top}\right]=\mathbf{0}$

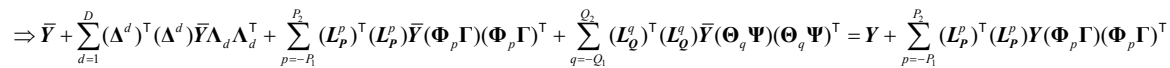

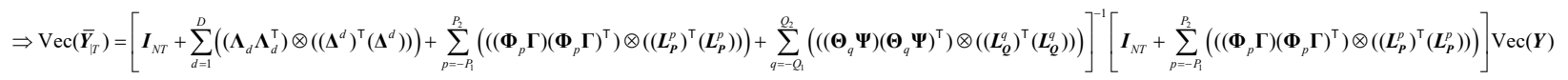

The sufficient second order condition for a unique global minimum at $\overline{\boldsymbol{Y}}_{\mid T}$ is satisfied:

$\frac{\partial^{2} S(\overline{\boldsymbol{Y}})}{\partial \overline{\boldsymbol{Y}} \boldsymbol{\overline { \boldsymbol { Y } }}}=-2 \frac{\partial}{\partial \operatorname{Vec}(\overline{\boldsymbol{Y}})^{\top}}\left[\operatorname{Vec}(\hat{\boldsymbol{Y}})-\sum_{d=1}^{D}\left(\left(\boldsymbol{\Lambda}_{d} \boldsymbol{\Lambda}_{d}^{\top}\right) \otimes\left(\left(\boldsymbol{\Delta}^{d}\right)^{\top}\left(\boldsymbol{\Delta}^{d}\right)\right)\right) \operatorname{Vec}(\overline{\boldsymbol{Y}})+\sum_{p=-p_{p}}^{p_{2}}\left(\left(\left(\boldsymbol{\Phi}_{p} \boldsymbol{\Gamma}\right)\left(\boldsymbol{\Phi}_{p} \boldsymbol{\Gamma}\right)^{\top}\right) \otimes\left(\left(\boldsymbol{(}_{p}^{p}\right)^{\top}\left(\boldsymbol{L}_{p}^{p}\right)\right)\right) \operatorname{Vec}(\hat{\boldsymbol{Y}})-\sum_{q=Q_{1}}^{Q_{2}}\left(\left(\left(\boldsymbol{\Theta}_{q} \boldsymbol{\Psi}\right)\left(\boldsymbol{\Theta}_{q} \boldsymbol{\Psi}^{\top}\right) \otimes\left(\left(\boldsymbol{L}_{Q}^{q}\right)^{\top}\left(\boldsymbol{L}_{Q}^{q}\right)\right)\right) \operatorname{Vec}(\overline{\boldsymbol{Y}})\right]\right.$ $=2\left[\boldsymbol{I}_{N T}+\sum_{d=1}^{D}\left(\left(\boldsymbol{\Lambda}_{d} \boldsymbol{\Lambda}_{d}^{\top}\right) \otimes\left(\left(\boldsymbol{\Delta}^{d}\right)^{\top}\left(\boldsymbol{\Delta}^{d}\right)\right)\right)+\sum_{p=-p_{1}}^{p_{3}}\left(\left(\left(\boldsymbol{\Phi}_{p} \boldsymbol{\Gamma}\right)\left(\boldsymbol{\Phi}_{p} \boldsymbol{\Gamma}\right)^{\top}\right) \otimes\left(\left(\boldsymbol{L}_{p}^{p}\right)^{\top}\left(\boldsymbol{L}_{p}^{p}\right)\right)\right)+\sum_{q=Q_{1}}^{O_{2}}\left(\left(\left(\boldsymbol{\Theta}_{q} \boldsymbol{\Psi}\right)\left(\boldsymbol{\Theta}_{q} \boldsymbol{\Psi}\right)^{\top}\right) \otimes\left(\left(\boldsymbol{L}_{Q}^{q}\right)^{\top}\left(\boldsymbol{L}_{Q}^{q}\right)\right)\right)\right]$

The Hessian matrix of the objective function $\frac{\partial^{2} S(\overline{\boldsymbol{Y}})}{\partial \overline{\boldsymbol{Y}} \partial \overline{\boldsymbol{Y}}^{\top}}$ is positive definite throughout its domain, because: i) the sum of positive definite and positive semidefinite matrices is positive definite; ii) the identity matrix is positive definite; iii) the Kronecker product of positive semidefinite matrices is positive semidefinite; and iv) the product of a matrix and its transpose is positive semidefinite. It follows that $\overline{\boldsymbol{Y}}_{\mid T}$ exists, because a positive definite matrix is nonsingular. 


\section{References}

Apel, M. and P. Jansson (1999), "System Estimates of Potential Output and the NAIRU", Empirical Economics, Vol. 24, pp. 373-388.

Calvo, G. (1983), "Staggered Prices in a Utility-Maximizing Framework”, Journal of Monetary Economics, Vol. 12, pp. 383-398.

Clark, P. (1989), "Trend Reversion in Real Output and Unemployment", Journal of Econometrics, Vol. 40, pp. 15-32.

de Jong, P. (1989), "Smoothing and Interpolation with the State-Space Model", Journal of the American Statistical Association, Vol. 84, pp. 1085-1088.

Galí, J. (2015), Monetary Policy, Inflation, and the Business Cycle: An Introduction to the New Keynesian Framework and Its Applications, Princeton University Press.

Hamilton, J. (1994), Time Series Analysis, Princeton University Press.

Hamilton, J. (2017), "Why You Should Never Use the Hodrick-Prescott Filter", NBER Working Paper, 23429.

Harvey, A. (1993), Time Series Models, MIT Press.

Harvey, A. and A. Jaeger (1993), "Detrending, Stylized Facts and the Business Cycle", Journal of Applied Econometrics, Vol. 8, pp. 231-247.

Hodrick, R. and E. Prescott (1997), "Post-War U.S. Business Cycles: A Descriptive Empirical Investigation”, Journal of Money, Credit and Banking, Vol. 29, pp. 1-16.

Kalman, R. (1960), "A New Approach to Linear Filtering and Prediction Problems", Transactions ASME Journal of Basic Engineering, Vol. 82, pp. 35-45.

King, R. and S. Rebelo (1993), "Low Frequency Filtering and Real Business Cycles", Journal of Economic Dynamics and Control, Vol. 17, pp. 207-231.

Laubach, T. and J. Williams (2003), "Measuring the Natural Rate of Interest", Review of Economics and Statistics, Vol. 85, pp. 1063-1070.

Laxton, D. and R. Tetlow (1992), “A Simple Multivariate Filter for the Measurement of Potential Output", Bank of Canada Technical Report, 59.

Lucas, R. (1980), "Two Illustrations of the Quantity Theory of Money", American Economic Review, Vol. 70, pp. 1005-1014.

Phillips, P. and S. Jin (2015), "Business Cycles, Trend Elimination, and the HP Filter", Cowles Foundation Discussion Paper, 2005.

Smets, F. and R. Wouters (2003), “An Estimated Dynamic Stochastic General Equilibrium Model of the Euro Area", Journal of the European Economic Association, Vol. 1, pp. 1123-1175.

Vitek, F. (2009), "Monetary Policy Analysis and Forecasting in the World Economy: A Panel Unobserved Components Approach", International Monetary Fund Working Paper, 238.

Woodford, M. (2003), Interest and Prices: Foundations of a Theory of Monetary Policy, Princeton University Press. 